\title{
Rigid automorphisms of linking systems
}

\author{
George Glauberman ${ }^{1}$ and Justin Lynd ${ }^{\left(\mathbb{D}_{2}\right.}$ \\ ${ }^{1}$ Department of Mathematics, University of Chicago, 5734 S. University Ave, Chicago, IL 60637; \\ E-mail: gg@math.uchicago.edu. \\ ${ }^{2}$ Department of Mathematics, University of Louisiana at Lafayette, Lafayette, LA 70504; E-mail: lynd@1ouisiana.edu.
}

Received: 13 May 2020; Revised: 15 January 2021; Accepted: 01 February 2021

2020 Mathematics Subject Classification: Primary - 20D20; Secondary - 20D45

\begin{abstract}
A rigid automorphism of a linking system is an automorphism that restricts to the identity on the Sylow subgroup. A rigid inner automorphism is conjugation by an element in the center of the Sylow subgroup. At odd primes, it is known that each rigid automorphism of a centric linking system is inner. We prove that the group of rigid outer automorphisms of a linking system at the prime 2 is elementary abelian and that it splits over the subgroup of rigid inner automorphisms. In a second result, we show that if an automorphism of a finite group $G$ restricts to the identity on the centric linking system for $G$, then it is of $p^{\prime}$-order modulo the group of inner automorphisms, provided $G$ has no nontrivial normal $p^{\prime}$-subgroups. We present two applications of this last result, one to tame fusion systems.
\end{abstract}

\section{Introduction}

A saturated fusion system $\mathcal{F}$ is a category in which the objects are the subgroups of a fixed finite $p$ group $S$ and the morphisms are injective group homomorphisms between subgroups, which are subject to axioms first outlined by Puig [Pui06, AKO11]. When $G$ is a finite group with Sylow $p$-subgroup $S$, there is a saturated fusion system $\mathcal{F}=\mathcal{F}_{S}(G)$ in which the morphisms are the $G$-conjugation maps between subgroups. One of the important properties of this category is that it keeps precisely the data required to recover the homotopy type of the Bousfield-Kan $p$-completion $B G_{p}^{\wedge}$ of the classifying space of $G$, as shown in the Martino-Priddy conjecture, proved by Oliver [Oli04, Oli06]. Recovery of $B G_{p}^{\wedge}$, or a $p$-complete space denoted $B \mathcal{F}$ when no group $G$ is associated with $\mathcal{F}$, is based on the construction of a centric linking system $\mathcal{L}$ for $\mathcal{F}$, an extension category of $\mathcal{F}$ whose existence and uniqueness up to rigid isomorphism was first established in general by Chermak [Che13]. From a group theoretic point of view, centric linking systems or, more generally, the transporter systems of Oliver-Ventura [OV07] and the localities of Chermak [Che13], provide finer approximations to $p$-local structure. They abstract the transporter categories of finite groups and form structures appearing in recent new approaches to revising the classification of finite simple groups.

We study here in more detail the comparison maps between automorphism groups of finite groups, linking systems and fusion systems. When $\mathcal{L}$ is a centric linking system associated to the fusion system $\mathcal{F}$, there are groups of automorphisms $\operatorname{Aut}(\mathcal{L})$ and $\operatorname{Aut}(\mathcal{F})$ and a map $\tilde{\mu}: \operatorname{Aut}(\mathcal{L}) \rightarrow \operatorname{Aut}(\mathcal{F})$ given essentially by restriction to the Sylow group $S$. When $\mathcal{L}=\mathcal{L}_{S}^{c}(G)$ and $\mathcal{F}=\mathcal{F}_{S}(G)$ for some finite group $G$, there is also a comparison map $\tilde{\kappa}_{G}: N_{\operatorname{Aut}(G)}(S) \rightarrow \operatorname{Aut}(\mathcal{L})$, where $N_{\text {Aut }(G)}(S)$ consists of those automorphisms of $G$ that leave $S$ invariant. These induce a pair 
of maps

$$
\operatorname{Out}(G) \stackrel{\kappa_{G}}{\longrightarrow} \operatorname{Out}(\mathcal{L}) \stackrel{\mu_{\mathcal{L}}}{\longrightarrow} \operatorname{Out}(\mathcal{F})
$$

on outer automorphism groups. We write $\operatorname{Aut}_{0}(\mathcal{L})$ for the group of rigid automorphisms of $\mathcal{L}$, namely, $\operatorname{ker}\left(\tilde{\mu}_{\mathcal{L}}\right)$. Similarly, $\operatorname{Out}_{0}(\mathcal{L})$ is short for $\operatorname{ker}\left(\mu_{\mathcal{L}}\right)$.

It follows from the exact sequence of [AKO11, III.5.12] and Chermak's theorem that $\mu_{\mathcal{L}}$ is an isomorphism if $p$ is odd and is surjective with kernel an abelian 2-group when $p=2$. Moreover, the surjectivity of $\kappa_{G}$ has been studied intensively in articles by Andersen, Oliver, and Ventura [AOV12] and by Broto, Møller, and Oliver [BMO19].

Our first result extends the consequences of unique existence of centric linking systems to show that the kernel of $\mu_{\mathcal{L}}$ is in fact of exponent at most 2, in general, when $p=2$. To make it easier to apply, we state and prove this in the slightly more general setting of a linking locality (defined just below) and in three equivalent ways. Set $k(p)=1$ if $p$ is odd and $k(p)=2$ if $p=2$. In particular, a group of exponent $k(p)$ is the trivial group if $p$ is odd and is elementary abelian if $p=2$.

Theorem 1.1 (Linking locality version). If $(\mathcal{L}, \Delta, S)$ is a linking locality at the prime $p$, then the group $\operatorname{Out}_{0}(\mathcal{L})$ of rigid outer automorphisms of $\mathcal{L}$ is abelian of exponent at most $k(p)$. Moreover, the exact sequence

$$
1 \rightarrow \operatorname{Aut}_{Z(S)}(\mathcal{L}) \rightarrow \operatorname{Aut}_{0}(\mathcal{L}) \rightarrow \operatorname{Out}_{0}(\mathcal{L}) \rightarrow 1
$$

splits.

Theorem 1.2 (Linking system version). If $\mathcal{L}$ is a linking system at the prime $p$ (in the general sense of [Hen19]), then the group $\operatorname{Out}_{0}(\mathcal{L})$ of rigid outer automorphisms of $\mathcal{L}$ is abelian of exponent at most $k(p)$. Moreover, the exact sequence

$$
1 \rightarrow \operatorname{Aut}_{Z(S)}(\mathcal{L}) \rightarrow \operatorname{Aut}_{0}(\mathcal{L}) \rightarrow \operatorname{Out}_{0}(\mathcal{L}) \rightarrow 1
$$

splits.

Theorem 1.3 (Cohomological version). Let $\mathcal{F}$ be a saturated fusion system over the finite p-group $S$, let $\mathcal{O}\left(\mathcal{F}^{c}\right)$ be the orbit category of $\mathcal{F}$-centric subgroups and let $\mathcal{Z}_{\mathcal{F}}: \mathcal{O}\left(\mathcal{F}^{c}\right)^{\mathrm{op}} \rightarrow$ Ab denote the center functor. Then $\lim ^{1} \mathcal{Z}_{\mathcal{F}}$ is of exponent at most $k(p)$. Moreover, the exact sequence

$$
1 \rightarrow \widehat{B}\left(\mathcal{O}\left(\mathcal{F}^{c}\right), \mathcal{Z}_{\mathcal{F}}\right) \rightarrow \widehat{Z}^{1}\left(\mathcal{O}\left(\mathcal{F}^{c}\right), \mathcal{Z}_{\mathcal{F}}\right) \rightarrow \lim ^{1} \mathcal{Z}_{\mathcal{F}} \rightarrow 1
$$

splits.

Here, a linking locality in the sense of [Hen19] (also called a proper locality in [Che15]) is a locality $(\mathcal{L}, \Delta, S)$ such that $\Delta$ contains all subgroups of $S$ that are centric and radical in $\mathcal{F}=\mathcal{F}_{S}(\mathcal{L})$, the fusion system of $\mathcal{L}$, and such that $C_{N_{\mathcal{L}}(P)}\left(O_{p}\left(N_{\mathcal{L}}(P)\right)\right) \leqslant O_{p}\left(N_{\mathcal{L}}(P)\right)$ for each $P \in \Delta$. Similarly, a linking system is a transporter system $\mathcal{L}$ associated with a saturated fusion system $\mathcal{F}$ such that $\operatorname{Ob}(\mathcal{L})$ contains all $\mathcal{F}$-centric radical subgroups and such that $C_{\text {Aut }_{\mathcal{L}}(P)}\left(O_{p}\left(\operatorname{Aut}_{\mathcal{L}}(P)\right)\right) \leqslant O_{p}\left(\operatorname{Aut}_{\mathcal{L}}(P)\right)$ for each $P \in \mathrm{Ob}(\mathcal{L})$. Other definitions of the term 'linking system' without further qualification, such as in [AKO11, Definition III.4.1], are special cases of this one.

An automorphism of a locality $\mathcal{L}$ is inner if it is induced by conjugation by an element of $N_{\mathcal{L}}(S)$, and a similar remark applies to transporter systems. In the case of a linking locality or linking system, a rigid inner automorphism is conjugation by an element of the center of $S$. We have denoted the group of rigid inner automorphisms by $\operatorname{Aut}_{Z(S)}(\mathcal{L})$. This helps to explain some of the terminology and notation in Theorems 1.1 and 1.2. We explain in more detail in Section 2. Terminology used in Theorem 1.3 is recalled in Section 3.

When $p$ is odd and $\mathcal{L}$ is a centric linking system, Theorems 1.1 to 1.3 follow from either of the alternative proofs of existence and uniqueness of centric linking systems as given in [Oli13] or 
[GL16]. The latter is based in part on the former but removes the dependence of the former on the classification of finite simple groups (CFSG). The connection between existence and uniqueness and the higher limits of the center functor $\mathcal{Z}_{\mathcal{F}}$ over the orbit category $\mathcal{O}\left(\mathcal{F}^{c}\right)$ of $\mathcal{F}$-centric subgroups is described by [AKO11, Proposition III.5.12]. In particular, this result identifies $\operatorname{Out}_{0}(\mathcal{L})$ with the first derived limit $\lim _{\mathcal{O}\left(\mathcal{F}^{c}\right)}^{1} \mathcal{Z}_{\mathcal{F}}$ of the center functor. So when $p$ is odd the theorems follow from [Oli13, Theorem 3.4] or [GL16, Theorem 1.1] and an argument, provided in Section 4, that uses Chermak's iterative procedure for extending a given locality to a new locality on a larger object set.

We shall prove Theorem 1.1 first in the case of a centric linking locality; that is, when $\Delta$ is the collection of $\mathcal{F}$-centric subgroups. The proof is applicable for all primes $p$, and so we obtain an alternative, somewhat simpler proof of the triviality of $\operatorname{Out}_{0}(\mathcal{L})$ for $p$ odd, independent of the main result of [GL16]. We then deduce Theorem 1.2 in the same special case, along with Theorem 1.3. Afterward, we shall prove in Section 4 that this implies the seemingly more general statements in Theorems 1.1 and 1.2.

Along the way, we extend to transporter systems a result of Oliver on isomorphisms of (quasicentric) linking systems (Proposition 2.5), and we interpret Chermak's work in the appendix of [Che13] as an equivalence of groupoids between localities and transporter systems (Theorem 2.11). Besides their use in deducing Theorem 1.2 from 1.1, one motivation for these extensions is to make clear that the results of [Oli13, GL16] give existence and uniqueness of centric linking localities up to rigid isomorphism in the same way as the main theorem of [Che13]. That this is not clear at first is caused by an ambiguity in which the notion of 'isomorphism' of a transporter system commonly in use does not restrict to the notion of 'automorphism' commonly in use but rather to what should be called 'rigid automorphism'.

Automorphisms of a finite group that centralise a Sylow subgroup have been studied by Glauberman, Gross, and others. The main result here can be seen as a generalisation to linking systems of [Gla68, Theorem 10]. The current work bears the same relationship to [Gla68, Theorem 10] as the proof of existence and uniqueness of centric linking systems outlined above does to the work of Gross [Gro82] and to the recent work of the authors with Guralnick and Navarro [GGLN20]. Our proof of Theorem 1.1 is very different from the proof of [Gla68, Theorem 10], however, in part because not all subgroups of $S$ need be objects.

Recall that for a finite group $G$ with Sylow $p$-subgroup $S$ and centric linking system $\mathcal{L}_{S}^{c}(G)$, there is a comparison homomorphism $\kappa_{G}: \operatorname{Out}(G) \rightarrow \operatorname{Out}\left(\mathcal{L}_{S}^{c}(G)\right)$. It is induced essentially by restriction to $p$-local structure modulo $p^{\prime}$-cores at the level of centric subgroups. In the course of trying to recover from the above theorems the corresponding results about finite groups, we were led to the following result, which seems to be of independent interest.

Theorem 1.4. Let $p$ be a prime and $G$ a finite group with Sylow p-subgroup $S$. If $O_{p^{\prime}}(G)=1$, then the kernel of the map $\kappa_{G}: \operatorname{Out}(G) \rightarrow \operatorname{Out}\left(\mathcal{L}_{S}^{c}(G)\right)$ is a $p^{\prime}$-group.

The proof of Theorem 1.4 relies on the $Z_{p}^{*}$-theorem, namely, the statement that an element $x \in S$ whose only $G$-conjugate in $S$ is $x$ itself must lie in the center of $G$ modulo $O_{p^{\prime}}(G)$. Thus, our proof of Theorem 1.4 depends on the CFSG if $p$ is odd. (This result and its corollaries in Section 5 for $p$ odd are the only results in the article that depend on the CFSG.)

When $G$ is simple, the cokernel of $\kappa_{G}$ has been studied extensively in [AOV12], [BMO19] and elsewhere. In particular, it has now been shown that the fusion system of each finite simple group $G$ is tame in the sense of [AOV12], namely, there is a possibly different finite group $G^{\prime}$ with Sylow subgroup $S$ such that $\mathcal{F}_{S}(G) \cong \mathcal{F}_{S}\left(G^{\prime}\right)$ such that the map $\kappa_{G^{\prime}}$ is split surjective. Theorem 1.4 has been shown in several special cases in the context of those works (cf. [BMO19, Lemma 5.9, Theorem 5.16]).

Theorem 1.4 is proved as Theorem 5.1 in Section 5, and we give two applications of it: We show that the splitting condition in the definition of a tame fusion system may be removed and we give an interesting reinterpretation of the first author's work on the Schreier conjecture [Gla66b]. 


\section{Terminology and notation}

When $G$ is a group and $g \in G$, we write $c_{g}$ for the left-handed conjugation homomorphism $x \mapsto g x g^{-1}$ and its restrictions. The image of a subgroup $P$ under $c_{g}$ is sometimes written in left-handed exponential notation ${ }^{g} P$. We write $\operatorname{Hom}_{G}(P, Q)$ for the set $\left\{c_{g} \mid g \in G,{ }^{g} P \leqslant Q\right\}$ of conjugation homomorphisms between $P$ and $Q$ induced in $G$. Given a finite group $G$ with Sylow $p$-subgroup $S$, the fusion system $\mathcal{F}_{S}(G)$ is the category with objects the subgroups of $S$ and with morphism sets $\operatorname{Hom}_{\mathcal{F}_{S}(G)}(P, Q):=$ $\operatorname{Hom}_{G}(P, Q):=\left\{c_{g} \mid g \in G,{ }^{g} P \leqslant Q\right\}$. Our terminology for fusion systems follows [AKO11]. For example, $\mathcal{F}^{c}$ denotes the set of $\mathcal{F}$-centric subgroups, $\mathcal{F}^{r}$ denotes the set of $\mathcal{F}$-radical subgroups, $\mathcal{F}^{f}$ denotes the set of fully $\mathcal{F}$-normalised subgroups and concatenation in the superscript denotes the intersection of the relevant sets.

\section{Transporter systems and localities}

Throughout this section, $\mathcal{F}$ is a saturated fusion system over a $p$-group $S$, and $\Delta$ is a nonempty collection of subgroups of $S$ that is closed under $\mathcal{F}$-conjugacy and passing to overgroups. Fix also another triple $\mathcal{F}^{\prime}, S^{\prime}$ and $\Delta^{\prime}$ of this type.

\subsection{Transporter systems}

In the case where $\mathcal{F}=\mathcal{F}_{S}(G)$ for some finite group $G$ with Sylow $p$-subgroup $S$, the transporter category $\mathcal{T}_{\Delta}(G)$ of $G$ with object set $\Delta$ is the category with morphisms $\operatorname{Mor}_{\mathcal{T}_{\Delta}(G)}(P, Q)=N_{G}(P, Q)=$ $\left\{g \in G \mid{ }^{g} P \leqslant Q\right\}$ where composition is given by multiplication in $G$. There is an inclusion functor $\delta: \mathcal{T}_{\Delta}(S) \rightarrow \mathcal{T}_{\Delta}(G)$, as well as a functor $\pi: \mathcal{T}_{\Delta}(G) \rightarrow \mathcal{F}_{S}(G)$, which is the inclusion on objects and which sends $g \in N_{G}(P, Q)$ to $c_{g} \in \operatorname{Hom}_{G}(P, Q)$, conjugation by $g$. This is the standard example of a transporter system associated with $\mathcal{F}_{S}(G)$.

Definition 2.1 ([OV07, Definition 3.1]). A transporter system associated with $\mathcal{F}$ is a nonempty category $\mathcal{T}$ with object set $\Delta \subseteq \operatorname{Ob}(\mathcal{F})$, together with structural functors

$$
\mathcal{T}_{\Delta}(S) \stackrel{\delta}{\rightarrow} \mathcal{T} \stackrel{\pi}{\rightarrow} \mathcal{F}
$$

that satisfy the following axioms:

(A1) $\Delta$ is closed under $\mathcal{F}$-conjugacy, and upon passing to overgroups, $\delta$ is the identity on objects and $\pi$ is the inclusion on objects.

(A2) For each $P, Q \in \Delta$, the kernel

$$
E(P):=\operatorname{ker}\left(\pi_{P, P}: \operatorname{Aut}_{\mathcal{T}}(P) \rightarrow \operatorname{Aut}_{\mathcal{F}}(P)\right)
$$

acts freely on $\operatorname{Mor}_{\mathcal{T}}(P, Q)$ by right composition, and $\pi_{P, Q}$ is the orbit map for this action. In particular, $\pi_{P, Q}$ is surjective. Also, $E(Q)$ acts freely on $\operatorname{Mor}_{\mathcal{T}}(P, Q)$ by left composition. Here, $\operatorname{Aut}_{\mathcal{T}}(P)$ denotes $^{\operatorname{Mor}_{\mathcal{T}}}(P, P)$.

(B) For each $P, Q \in \Delta, \delta_{P, Q}: N_{S}(P, Q) \rightarrow \operatorname{Mor}_{\mathcal{T}}(P, Q)$ is injective, and the composite $\pi_{P, Q} \circ \delta_{P, Q}$ sends $g \in N_{S}(P, Q)$ to $c_{g} \in \operatorname{Hom}_{\mathcal{F}}(P, Q)$.

(C) For each $\varphi \in \operatorname{Mor}_{\mathcal{T}}(P, Q)$ and each $g \in P$, the diagram

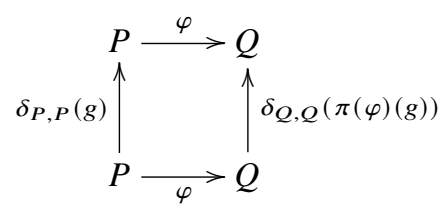

commutes in $\mathcal{T}$. 
(I) $\delta_{S, S}(S)$ is a Sylow $p$-subgroup of $\operatorname{Aut}_{\mathcal{T}}(S)$.

(II) Let $\varphi \in \operatorname{Iso}_{\mathcal{T}}(P, Q), P \triangleleft \bar{P} \leqslant S$ and $Q \triangleleft \bar{Q} \leqslant S$ be such that $\varphi \circ \delta_{P, P}(\bar{P}) \circ \varphi^{-1} \leqslant \delta_{Q, Q}(\bar{Q})$. Then there is $\bar{\varphi} \in \operatorname{Mor}_{\mathcal{T}}(\bar{P}, \bar{Q})$ such that $\bar{\varphi} \circ \delta_{P, \bar{P}}(1)=\delta_{Q, \bar{Q}}(1) \circ \varphi$.

From now on, we abbreviate $\delta_{P, P}$ to $\delta_{P}, \pi_{P, P}$ to $\pi_{P}$ and use similar notation when considering the application of an arbitrary functor on morphism sets. In addition, any future reference to axioms (A1)-(II) should be interpreted as reference to the axioms given in Definition 2.1. The following lemma collects some basic properties of morphisms in a transporter system.

Lemma 2.2. Fix a transporter system $(\mathcal{T}, \delta, \pi)$ associated with $\mathcal{F}$.

(a) Each morphism in $\mathcal{T}$ is both a monomorphism and an epimorphism in the categorical sense.

(b) (Restrictions are unique) Given objects $P_{0} \leqslant P, Q_{0} \leqslant Q$ and two morphisms $\varphi_{0}$, $\varphi_{0}^{\prime}$ making the diagram

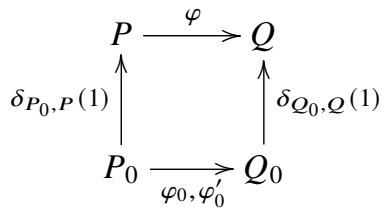

commute, one has $\varphi_{0}=\varphi_{0}^{\prime}$.

(c) (Extensions are unique) Given objects $P_{0} \leqslant P, Q_{0} \leqslant Q$ and two morphisms $\varphi$, $\varphi^{\prime}$ making the diagram

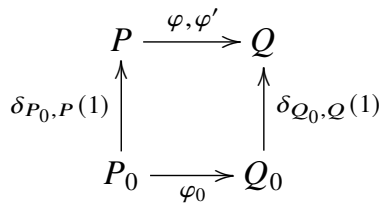

commute, one has $\varphi=\varphi^{\prime}$.

Proof. Parts (a) and (b) are contained in [OV07, Lemma 3.2], and part (c) is proved in [Che13, Lemma A.5(c)].

A morphism of fusion systems $\mathcal{F} \rightarrow \mathcal{F}^{\prime}$ means a pair $(\alpha, \Phi)$ where $\alpha: S \rightarrow S^{\prime}$ is a group homomorphism and $\Phi: \mathcal{F} \rightarrow \mathcal{F}^{\prime}$ is a functor, which together satisfy $\alpha(P)=\Phi(P)$ on objects and $\Phi(\varphi) \circ \alpha=\alpha \circ \varphi$ for each morphism $\varphi$ in $\mathcal{F}$. If $\alpha$ is an isomorphism, then $\Phi$ is determined uniquely by $\alpha$. So an isomorphism of fusion systems may be regarded as an isomorphism of the underlying $p$-groups that 'preserves fusion'.

Definition 2.3 (Isomorphisms of transporter systems). Let $(\mathcal{T}, \delta, \pi)$ and $\left(\mathcal{T}, \delta^{\prime}, \pi^{\prime}\right)$ be transporter systems with object sets $\Delta$ and $\Delta^{\prime}$ for the saturated fusion systems $\mathcal{F}$ and $\mathcal{F}^{\prime}$, respectively.

1. Let $\alpha: \mathcal{T} \rightarrow \mathcal{T}$ be an equivalence of categories. It is said that

$\circ \alpha$ is isotypical if $\alpha\left(\delta_{P}(P)\right)=\delta_{\alpha(P)}^{\prime}(\alpha(P))$ for each subgroup $P \in \Delta$ and

○ $\alpha$ sends inclusions to inclusions if $\alpha\left(\delta_{P, Q}(1)\right)=\delta_{\alpha(P), \alpha(Q)}^{\prime}(1)$ for each $P, Q \in \Delta$.

2. An isomorphism is an equivalence $\mathcal{T} \rightarrow \mathcal{T}$ that is isotypical and sends inclusions to inclusions. An automorphism is an isomorphism of a transporter system onto itself.

3. An isomorphism $\alpha: \mathcal{T} \rightarrow \mathcal{T}$ is said to be rigid if $S=S^{\prime}$ and $\alpha_{S} \circ \delta_{S}=\delta_{S}^{\prime}$ as homomorphisms $S \rightarrow \operatorname{Aut}_{\mathcal{T}^{\prime}}(S)$. Here, as before, $\alpha_{S}$ means $\alpha_{S, S}$.

4. An automorphism $\alpha$ of $\mathcal{T}$ is inner if there is an element $\varphi \in \operatorname{Aut}_{\mathcal{T}}(S)$ such that $\alpha$ is given on objects by $P \mapsto c_{\varphi}(P):=\pi(\varphi)(P)$ and on morphisms by mapping $\psi: P \rightarrow Q$ to

$$
c_{\varphi}(\psi):=\left.\varphi\right|_{Q, c_{\varphi}(Q)} \circ \psi \circ\left(\left.\varphi\right|_{P, c_{\varphi}(P)}\right)^{-1},
$$


where, for example, $\left.\varphi\right|_{Q, c_{\varphi}(Q)}$ is the unique morphism from $Q$ to $c_{\varphi}(Q)$ in $\mathcal{T}$ such that $\varphi \circ \delta_{Q, S}(1)=$ $\delta_{c_{\varphi}(Q), S}(1) \circ \varphi$, as given by Lemma 2.2(b). We refer to $c_{\varphi}$ as conjugation by $\varphi$. Write $\operatorname{Aut}_{Z(S)}(\mathcal{T})$ for the group of rigid inner automorphisms of $\mathcal{T}$ that are conjugation by elements of $\delta_{S}(Z(S)) \leqslant$ Aut $\mathcal{T}(S)$.

Denote by $\operatorname{Aut}(\mathcal{T}):=\operatorname{Aut}(\mathcal{T}, \delta, \pi)$ the group of automorphisms of $\mathcal{T}$. Denote by $\mathrm{T}$ the category of transporter systems and isomorphisms.

Remark 2.4. An isomorphism of transporter systems is in particular an invertible functor, and so one sees that $\operatorname{Aut}(\mathcal{T})$ is indeed a group. This was shown for linking systems in [AOV12, Lemma 1.14(a)], and the same argument applies for an arbitrary transporter system.

We have defined isomorphism here in analogy with the definition of an automorphism of a centric linking system [AKO11, III.4.3] but more generally than is usually done. The usual definition of an isomorphism of transporter systems is a functor $\alpha: \mathcal{T} \rightarrow \mathcal{T}$ that commutes with the structural functors $\alpha \circ \delta=\delta^{\prime}$ and $\pi^{\prime} \circ \alpha=\pi$. See, for example, [BLO03, p.799], [OV07, Proposition 3.11], [AKO11, p.146] or [Che13, Definition A.2]. Rather, Definition 2.3 specialises to the definition of an automorphism of a linking system in [AKO11, Section III.4.3].

The following proposition extends Proposition 4.11 of [AKO11] in two ways, but the proof follows the same basic outline. It helps explain that an isomorphism between transporter systems is equivalent to a triple of functors commuting with the structural functors and that the usual definition of isomorphism of transporter systems is the same as what we are calling a rigid isomorphism.

Proposition 2.5. Fix transporter systems $(\mathcal{T}, \delta, \pi)$ and $\left(\mathcal{T}, \delta^{\prime}, \pi^{\prime}\right)$ associated to $\mathcal{F}$ and $\mathcal{F}^{\prime}$ with object sets $\Delta$ and $\Delta^{\prime}$ that contain $\mathcal{F}^{c r}$ and $\mathcal{F}^{\prime c r}$. Given an isomorphism $\alpha: \mathcal{T} \rightarrow \mathcal{T}$ in the sense of Definition 2.3, there is a unique associated isomorphism $\beta: S \rightarrow S^{\prime}$, a unique functor $\beta_{*}: \mathcal{T}_{\Delta}(S) \rightarrow \mathcal{T}_{\Delta^{\prime}}\left(S^{\prime}\right)$ and a unique isomorphism $c_{\beta}:: \mathcal{F} \rightarrow \mathcal{F}^{\prime}$ of fusion systems such that the diagram

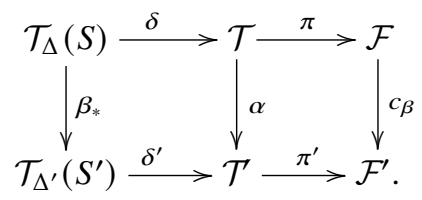

commutes and $\beta=\left(\beta_{*}\right)_{S}$. Moreover, $\alpha$ is a rigid isomorphism if and only if both $\beta_{*}$ and $c_{\beta}$ are the identity functors.

Proof. Let $\alpha: \mathcal{T} \rightarrow \mathcal{T}$ be an isomorphism. Because $S$ is the only object of $\mathcal{T}$ with the property that $\operatorname{Mor}_{\mathcal{T}}(P, S) \neq \varnothing$ for each object $P$ of $\mathcal{T}$, and the same is true for $S^{\prime}$ with respect to $\mathcal{T}$, it follows that $\alpha(S)=S^{\prime}$. So $\alpha_{S}\left(\delta_{S}(S)\right)=\delta_{S^{\prime}}^{\prime}\left(S^{\prime}\right)$ because $\alpha$ is isotypical. By axiom (B) for a transporter system, the map $\delta_{S^{\prime}}^{\prime}: S^{\prime} \rightarrow \delta_{S^{\prime}}^{\prime}\left(S^{\prime}\right)$ is an isomorphism, so there is a unique map $\beta$ from $S=\operatorname{Aut}_{\mathcal{T}_{\Delta}(S)}(S)$ to $S^{\prime}=\operatorname{Aut}_{\mathcal{T}_{\Delta^{\prime}}\left(S^{\prime}\right)}\left(S^{\prime}\right)$ such that

$$
\alpha_{S}\left(\delta_{S}(s)\right)=\delta_{S^{\prime}}^{\prime}(\beta(s))
$$

for each $s \in S$. Then $\beta=\left(\delta^{\prime}\right)_{S^{\prime}}^{-1} \circ \alpha_{S} \circ \delta_{S}$ is an isomorphism from $S$ to $S^{\prime}$. Now $\alpha$ sends inclusions to inclusions and so commutes with restrictions. Hence, for each $P \in \Delta$, as $\alpha\left(\delta_{P}(P)\right)=\delta_{\alpha(P)}^{\prime}(\alpha(P))$, we have $\alpha_{S}\left(\delta_{S}(P)\right)=\delta_{S^{\prime}}^{\prime}(\alpha(P))$, and this shows with (2.7) and injectivity of $\delta^{\prime}$ that $\beta(P)=\alpha(P)$ for each $P$.

Let $\beta_{*}: \mathcal{T}_{\Delta}(S) \rightarrow \mathcal{T}_{\Delta^{\prime}}\left(S^{\prime}\right)$ be the functor induced by $\beta$. Namely, $\beta_{*}$ sends an object $P$ to $\beta(P)$, and it sends a morphism $P \stackrel{s}{\rightarrow} Q$ to $\beta(P) \stackrel{\beta(s)}{\rightarrow} \beta(Q)$. Then $\delta^{\prime} \circ \beta_{*}=\alpha \circ \delta$ by construction.

Next, we wish to define a functor $c_{\beta}: \mathcal{F} \rightarrow \mathcal{F}^{\prime}$ via a mapping on objects sending $P$ to $\beta(P)$ and on morphisms sending $P \stackrel{\varphi}{\rightarrow} Q$ to $\beta(P) \stackrel{\beta \circ \varphi \circ \beta^{-1}}{\longrightarrow} \beta(Q)$. This is an isomorphism of fusion systems (the one corresponding to the isomorphism $\beta$ from $S$ to $S^{\prime}$ ) with inverse $c_{\beta^{-1}}$, if well defined. In order to show 
that the assignment is well defined, we must prove that each $\beta \circ \varphi \circ \beta^{-1}$ is a morphism in $\mathcal{F}^{\prime}$. This will be done by showing that $c_{\beta}(\varphi)=\pi^{\prime}(\alpha(\tilde{\varphi}))$ for each $\tilde{\varphi} \in \operatorname{Mor}_{\mathcal{T}}(P, Q)$ with $\pi(\tilde{\varphi})=\varphi$, thus simultaneously showing that the right square in (2.6) commutes.

Fix such a lift $\tilde{\varphi}$ of $\varphi$, and let $s \in P$. Consider the following diagrams:

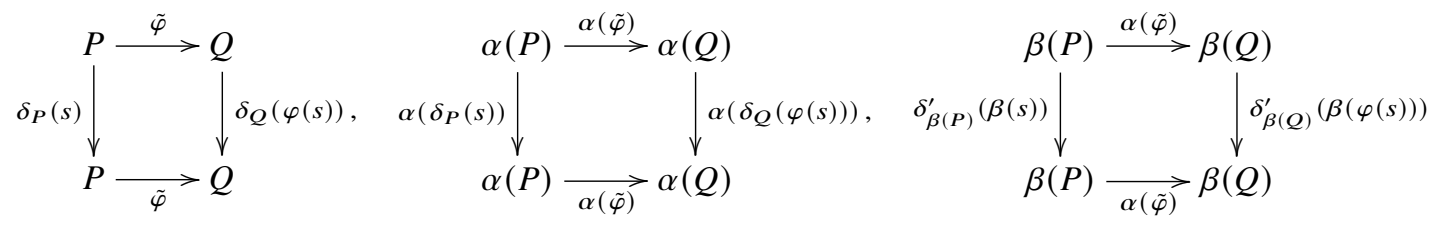

By axiom (C) for $\mathcal{T}$, the first diagram commutes, and the second is $\alpha$ applied to the first. As shown above, $\beta(P)=\alpha(P)$ and $\alpha \circ \delta=\delta^{\prime} \circ \beta_{*}$, so the third diagram is the same as the second. By axiom (C) for $\mathcal{T}$ with $\alpha(\tilde{\varphi})$ and $\beta(s)$ in the roles of $\varphi$ and $g$, the morphism $\delta_{\beta(Q)}^{\prime}\left(\pi^{\prime}(\alpha(\tilde{\varphi}))(\beta(s))\right)$ in place of $\delta_{\beta(Q)}^{\prime}(\beta(\varphi(s)))$ also makes the third diagram commute, so we have

$$
\delta_{\beta(Q)}^{\prime}(\beta(\varphi(s))) \circ \alpha(\tilde{\varphi})=\delta_{\beta(Q)}^{\prime}\left(\pi^{\prime}(\alpha(\tilde{\varphi}))(\beta(s))\right) \circ \alpha(\tilde{\varphi})
$$

as morphisms between $\beta(P)$ and $\beta(Q)$ in $\mathcal{T}$. Because each morphism in a transporter system is an epimorphism (Lemma 2.2(a)) and $\delta_{\beta(Q)}^{\prime}$ is injective (axiom (B)), it follows that

$$
\beta(\varphi(s))=\pi^{\prime}(\alpha(\tilde{\varphi}))(\beta(s)), \quad \text { for } s \in P .
$$

Hence, after replacing $s$ by $\beta^{-1}(s)$, we see that $c_{\beta}(\varphi)=\pi^{\prime}(\alpha(\tilde{\varphi}))$ as claimed, and this completes the proof of existence of the functors $\beta_{*}$ and $c_{\beta}$.

It remains to prove uniqueness. Observe that uniqueness of $\beta$ would follow from that of $\beta_{*}$. Suppose $\gamma: \mathcal{T}_{\Delta}(S) \rightarrow \mathcal{T}_{\Delta^{\prime}}\left(S^{\prime}\right)$ is a functor such that $\gamma$ in place of $\beta^{*}$ makes the left square in (2.6) commute. Because $\delta$ and $\delta^{\prime}$ are the identity on objects by axiom (A1), $\gamma$ agrees with $\beta_{*}$ on objects. Similarly, they agree on morphisms, given commutativity of the diagram, because $\delta^{\prime}{ }_{P, Q}$ is injective by axiom (B) for each $P, Q \in \Delta$. Hence, $\gamma=\beta_{*}$. Next, suppose in addition that $\eta: \mathcal{F} \rightarrow \mathcal{F}^{\prime}$ is another functor such that the right square in (2.6) commutes with $\eta$ in place of $c_{\beta}$. By axiom (A1), the functors $c_{\beta}$ and $\eta$ agree with $\alpha$ on the objects $\Delta$. For each morphism $\varphi$ in $\mathcal{T}$ between subgroups in $\Delta$, we have $\eta(\pi(\varphi))=c_{\beta}(\pi(\varphi))$, so by axiom (A2) on the surjectivity of $\pi$ on morphism sets we see that $\eta$ and $c_{\beta}$ agree on morphisms in $\mathcal{F}$ between subgroups in $\Delta$. By assumption $\mathcal{F}^{c r} \subseteq \Delta$, the Alperin-Goldschmidt fusion theorem [BLO03, Proposition A.10] or [AKO11, I.3.5] gives equality.

If $\alpha$ is a rigid isomorphism, then by definition $S=S^{\prime}$. By commutativity of the left square in (2.6), $\delta_{S}^{\prime} \circ \beta=\alpha_{S} \circ \delta_{S}=\delta_{S}^{\prime}$. So $\beta=\mathrm{id}_{S}$ as $\delta_{S}^{\prime}$ is injective. It was shown above that $\beta_{*}$ and $c_{\beta}$ are uniquely determined by $\beta$, so $\beta_{*}$ and $c_{\beta}$ are the identity. Conversely, if $\beta_{*}$ is the identity functor, then $S=S^{\prime}$, and by commutativity of the left square we have $\alpha_{S} \circ \delta_{S}=\delta_{S}^{\prime} \circ \operatorname{id}_{S}=\delta_{S}^{\prime}$, so $\alpha$ is rigid.

As in the setting of (quasicentric) linking systems [AOV12, p.197], one can define a group homomorphism relating automorphisms of a transporter system with automorphisms of the associated fusion system in this more general setting using Proposition 2.5. Let $(\mathcal{T}, \delta, \pi)$ be a transporter system with object set $\Delta$ associated with the saturated fusion system $\mathcal{F}$ on $S$. Assume that $\mathcal{F}^{c r} \subseteq \Delta$. Define

$$
\tilde{\mu}_{\mathcal{T}}: \operatorname{Aut}(\mathcal{T}) \rightarrow \operatorname{Aut}(\mathcal{F})
$$

to be the map that sends $\alpha \in \operatorname{Aut}(\mathcal{T})$ to the automorphism $\delta_{S}^{-1} \circ \alpha_{S} \circ \delta_{S}$ of $S=\operatorname{Aut}_{\mathcal{T}_{\Delta}(S)}(S)$. Thus, $\tilde{\mu}_{\mathcal{T}}(\alpha)$ is the automorphism $\beta$ in Proposition 2.5. This is a group homomorphism (using uniqueness of $c_{\beta}$ ) that maps $\operatorname{Aut}_{\mathcal{T}}(S)$ onto $\operatorname{Aut}_{\mathcal{F}}(S)$ and has kernel $\operatorname{Aut}_{0}(\mathcal{T})$. It induces a homomorphism

$$
\mu_{\mathcal{T}}: \operatorname{Out}(\mathcal{T}) \rightarrow \operatorname{Out}(\mathcal{F})
$$


with kernel Out $0(\mathcal{T})$. When $\mathcal{T}=\mathcal{T}_{\Delta}(G)$ for some finite group $G$ with Sylow $p$-subgroup $S$, we sometimes write $\tilde{\mu}_{G}$ for $\tilde{\mu}_{\mathcal{T}}$ and $\mu_{G}$ for $\mu_{\mathcal{T}}$, provided $\mathcal{T}$ is understood from the context.

\subsection{Localities}

In his proof of the existence and uniqueness of centric linking systems, Chermak introduced localities and showed in [Che13, Appendix] that they are essentially equivalent to transporter systems. The purpose of this subsection is to explain how Chermak's results give an equivalence of categories between transporter systems and localities, with morphisms isomorphisms, while setting up notation.

Let $\mathcal{L}$ be a finite set (we shall consider only finite localities). Write $\mathbf{W}(\mathcal{L})$ for the monoid of words $\left(f_{n}, \ldots, f_{1}\right)$ in the elements of $\mathcal{L}$, where the multiplication is concatenation $\circ$. A partial group is a set $\mathcal{L}$ together with a subset $\mathbf{D}:=\mathbf{D}(\mathcal{L}) \subseteq \mathbf{W}(\mathcal{L})$, a multivariable product $\Pi: \mathbf{D} \rightarrow \mathcal{L}$ defined on words in $\mathbf{D}$ and an inversion map $(-)^{-1}: \mathcal{L} \rightarrow \mathcal{L}$, subject to certain axioms that may be found in [Che13, Definition 2.1]. The product $f_{n} \cdots f_{1}$ is defined if $\left(f_{n}, \ldots, f_{1}\right) \in \mathbf{D}$, and in this case we set $f_{n} \cdots f_{1}=\Pi\left(f_{n}, \ldots, f_{1}\right)$. A partial group is a group if and only if $\mathbf{D}=\mathbf{W}(\mathcal{L})$; that is, all products are defined. A partial subgroup is a subset $\mathcal{L}_{0}$ of $\mathcal{L}$ with domain $\mathbf{D}_{0} \subseteq \mathbf{W}\left(\mathcal{L}_{0}\right) \cap \mathbf{D}$, such that the restriction of the product $\Pi$ to $\mathbf{D}_{0}$ is the product $\Pi_{0}$ for $\mathcal{L}_{0}$. The subgroups of $\mathcal{L}$ are the partial subgroups $\mathcal{L}_{0}$ with $\mathbf{W}\left(\mathcal{L}_{0}\right) \subseteq \mathbf{D}(\mathcal{L})$. A homomorphism of partial groups is a function $\gamma: \mathcal{L} \rightarrow \mathcal{M}$ such that $\gamma^{*}(\mathbf{D}(\mathcal{L})) \subseteq \mathbf{D}(\mathcal{M})$ and $\Pi\left(\gamma^{*}(w)\right)=\gamma(\Pi(w))$ for any word $w \in \mathbf{D}(\mathcal{L})$. Here, $\gamma^{*}: \mathbf{W}(\mathcal{L}) \rightarrow \mathbf{W}(\mathcal{M})$ is the map on words determined by $\gamma$. Partial groups and partial group homomorphisms form a category, so there is the usual notion of isomorphism in this category. A homomorphism $\gamma$ as above is an isomorphism if and only if it is a bijective homomorphism satisfying $\gamma^{*}(\mathbf{D}(\mathcal{L}))=\mathbf{D}(\mathcal{M})$.

There is a natural notion of conjugation in a partial group when defined. Given $f \in \mathcal{L}$, write $\mathbf{D}(f)$ for the set of $x \in \mathcal{L}$ such that $\left(f, x, f^{-1}\right) \in \mathbf{D}$. The product $f x f^{-1}=\Pi\left(f, x, f^{-1}\right)$ is the conjugate of $x$ by $f$, sometimes written ${ }^{f} x$. A usual convention, which we adopt, is that any such expression carries the tacit assumption that $x \in \mathbf{D}(f)$. Likewise, for any subset $X \subseteq \mathcal{L}$, the expression ${ }^{f} X$ has a similar meaning, including that $X \subseteq \mathbf{D}(f)$.

Definition 2.8. Let $\mathcal{L}$ be a finite partial group, let $S$ be a $p$-subgroup of $\mathcal{L}$ and let $\Delta$ be a collection of subgroups of $S$. The triple $(\mathcal{L}, \Delta, S)$ is a locality if

(L1a) $\mathbf{D}(\mathcal{L})$ is equal to the set of those $\left(f_{n}, \ldots, f_{1}\right) \in \mathbf{W}(\mathcal{L})$ such that there is $\left(X_{0}, \ldots, X_{n}\right) \in \mathbf{W}(\Delta)$ with ${ }^{f_{i+1}} X_{i}=X_{i+1}$ for each $0 \leqslant i<n$.

(L1b) If $P \in \Delta$ and $f \in \mathcal{L}$ with $P \leqslant \mathbf{D}(f)$ and ${ }^{f} P \leqslant S$, then $Q \in \Delta$ for each ${ }^{f} P \leqslant Q \leqslant S$.

(L2) $S$ is a maximal member of the poset of $p$-subgroups of $\mathcal{L}$.

We next set up some notation when working with a locality $(\mathcal{L}, \Delta, S)$. A word $w=\left(f_{n}, \ldots, f_{1}\right) \in$ $\mathbf{W}(\mathcal{L})$ is in $\mathbf{D}(\mathcal{L})$ via $X_{0}$ if $f_{i} \cdots f_{1} X_{0} \in \Delta$ for each $1 \leqslant i \leqslant n$; compare (L1a). For $f \in \mathcal{L}$, denote by $S_{f}$ the set of $s \in S$ such that ${ }^{f} s \in S$. By [Che13, Proposition 2.11], $S_{f} \in \Delta$. In particular, $S_{f}$ is a subgroup of $\mathcal{L}$ that plays the role of a Sylow intersection. For an object $P \in \Delta$, the normaliser $N_{\mathcal{L}}(P)=\left\{f \in \mathcal{L} \mid{ }^{f} P=P\right\}$ and centraliser $C_{\mathcal{L}}(P)=\left\{f \in \mathcal{L} \mid{ }^{f} x=x\right.$ for all $\left.x \in P\right\}$ are subgroups of $\mathcal{L}$.

The fusion system $\mathcal{F}_{S}(\mathcal{L})$ of $\mathcal{L}$ is the fusion system on $S$ with morphisms being those group monomorphisms between subgroups of $S$ that can be written as compositions of restrictions of the conjugation homomorphisms $c_{f}: P \rightarrow Q, x \mapsto{ }^{f} x$ between objects $P, Q \in \Delta$. It is said that $\mathcal{L}$ is a locality on $\mathcal{F}_{S}(\mathcal{L})$.

Example 2.9 ([Che13, Example/Lemma 2.10]). Let $G$ be a finite group, let $S$ be a Sylow $p$-subgroup of $G$ and let $\Delta$ be a collection of subgroups of $S$ that is closed under $\mathcal{F}_{S}(G)$-conjugacy and upon passing to overgroups and that contains all $\mathcal{F}_{S}(G)$-centric radical subgroups. Let $\mathcal{L}$ be the subset of $G$ consisting of those $g \in G$ such that there exists $P \in \Delta$ with ${ }^{g} P \leqslant S$ (so that ${ }^{g} P \in \Delta$ ). Let $\mathbf{D} \subseteq \mathbf{W}(\mathcal{L})$ denote the collection of all words $\left(g_{n}, \ldots, g_{1}\right) \in \mathbf{W}(\mathcal{L})$ such that there is $\left(X_{0}, \ldots, X_{n}\right) \in \mathbf{W}(\Delta)$ with ${ }^{g_{i} \cdots g_{1}} X_{0} \in \Delta$ for each $0 \leqslant i \leqslant n$. Whenever $\left(g_{n}, \ldots, g_{1}\right)$ is a word in $\mathbf{D}$, define $\Pi\left(g_{n}, \ldots, g_{1}\right)=g_{n} \cdots g_{1}$, the product in $G$. Then $(\mathcal{L}, \Delta, S)$ is a locality on $\mathcal{F}_{S}(G)$, written $\mathcal{L}_{\Delta}(G)$. 
Definition 2.10 (Isomorphisms of localities). Let $(\mathcal{L}, \Delta, S)$ and $\left(\mathcal{L}^{\prime}, \Delta^{\prime}, S^{\prime}\right)$ be localities.

1. An isomorphism from $(\mathcal{L}, \Delta, S)$ to $\left(\mathcal{L}^{\prime}, \Delta^{\prime}, S^{\prime}\right)$ is an isomorphism of partial groups $\beta: \mathcal{L} \rightarrow \mathcal{L}^{\prime}$ such that $\beta(\Delta)=\Delta^{\prime}$ (hence, $\left.\beta(S)=S^{\prime}\right)$. An automorphism of $(\mathcal{L}, \Delta, S)$ is an isomorphism of $(\mathcal{L}, \Delta, S)$ to itself.

2. An isomorphism $\beta$ is rigid if $S=S^{\prime}$, and $\beta$ is the identity on $S$.

3. An automorphism $\alpha$ of $\mathcal{L}$ is inner if it is given by conjugation by an element of $N_{\mathcal{L}}(S)$, namely, there is $f \in N_{\mathcal{L}}(S)$ such that $\alpha(x)=f x f^{-1}$ for all $x \in \mathcal{L}$. (Note that the product $f x f^{-1}$ is always defined when $f \in N_{\mathcal{L}}(S)$.)

Write $\operatorname{Aut}(\mathcal{L}):=\operatorname{Aut}(\mathcal{L}, \Delta, S)$ for the group of automorphisms of $\mathcal{L}, \operatorname{Aut}_{0}(\mathcal{L})$ for the subgroup of rigid automorphisms and $\operatorname{Aut}_{Z(S)}(\mathcal{L})$ for the subgroup of $\operatorname{Aut}_{0}(\mathcal{L})$ consisting of automorphisms that are conjugation by elements in $Z(S)$. Denote by $L$ the category of localities with isomorphisms.

\subsection{Equivalence between transporter systems and localities}

In [Che13, Appendix], Chermak goes most of the way toward proving that there is an equivalence between the category of transporter systems with rigid isomorphisms (in the sense of Definition 2.3) and the category of localities with rigid isomorphisms. Here, we suggest a mild extension of Chermak's results to an equivalence of the slightly larger categories $T$ and $L$ with the same objects. First, we briefly review how to pass from a locality to a transporter system and vice versa. More details are given in [Che13, Appendix A].

\subsubsection{From localities to transporter systems}

Given a locality $(\mathcal{L}, \Delta, S)$, one can make a transporter system $\left(\mathcal{T}_{\Delta}(\mathcal{L}), \delta, \pi\right)$ associated with $\mathcal{F}_{S}(\mathcal{L})$ in the following way. Let $\mathcal{T}_{\Delta}(\mathcal{L})$ have object set $\Delta$, and for each $P, Q \in \Delta$ take

$$
\operatorname{Mor}_{\mathcal{T}_{\Delta}(\mathcal{L})}(P, Q)=\left\{(f, P, Q) \mid f \in \mathcal{L},{ }^{f} P \leqslant Q\right\}
$$

Composition is given by multiplication in $\mathcal{L}$. The functor $\delta$ is the identity on objects and sends $P \stackrel{s}{\rightarrow} Q$ to $(s, P, Q)$. The functor $\pi$ is the inclusion on objects and sends $(f, P, Q)$ to the conjugation homomorphism $c_{f}: P \rightarrow Q$.

\subsubsection{From transporter systems to localities}

Conversely, to make a locality given a transporter system $(\mathcal{T}, \delta, \pi)$, consider the collection of isomorphisms Iso $(\mathcal{T})$ in $\mathcal{T}$ and the following relation on the set $\operatorname{Mor}(\mathcal{T})$ of morphisms in $\mathcal{T}$ : the morphism $\varphi: P \rightarrow Q$ is an extension of $\varphi_{0}: P_{0} \rightarrow Q_{0}$, written $\varphi_{0} \uparrow \varphi$, if the diagram

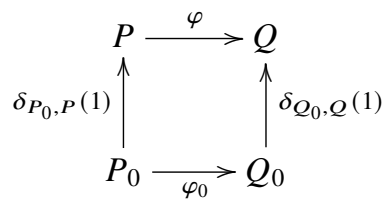

commutes in $\mathcal{T}$. This is a partial order, and the equivalence relation on $\operatorname{Iso}(\mathcal{T})$ generated by its restriction to $\operatorname{Iso}(\mathcal{T})$ is denoted $\equiv$. It is shown in [Che13, Lemma A.8(a)] that each $\equiv$-class has a unique maximal member with respect to $\uparrow$. Write $[\varphi]$ for the equivalence class of $\varphi$, and $\operatorname{set}(\mathcal{L}, \Delta, S)=(\operatorname{Iso}(\mathcal{T}) / \equiv, \Delta, S)$, where, by abuse of notation, $S$ is identified with the set of equivalence classes $\left\{\left[\delta_{S}(s)\right] \mid s \in S\right\}$ of elements in $\delta_{S}(S) \subseteq \operatorname{Aut}_{\mathcal{T}}(S) \subseteq \operatorname{Iso}(\mathcal{T})$. The domain $\mathbf{D}\left(\mathcal{L}_{\Delta}(\mathcal{T})\right)$ for the product is the set of all words $\left(f_{n}, \ldots, f_{1}\right) \in \mathbf{W}\left(\mathcal{L}_{\Delta}(\mathcal{T})\right)$ such that there exist objects $P_{0}, \ldots, P_{n} \in \Delta$ and isomorphisms $\varphi_{i}: P_{i-1} \rightarrow P_{i}$ in $\mathcal{T}$ such that $\varphi_{i} \in f_{i}$ for each $i$. The product $\Pi: \mathbf{D}\left(\mathcal{L}_{\Delta}(\mathcal{T})\right) \rightarrow \mathcal{L}_{\Delta}(\mathcal{T})$ is defined by $\Pi\left(f_{n}, \ldots, f_{1}\right)=\left[\varphi_{n} \circ \cdots \circ \varphi_{1}\right]$. The inversion map $-^{-1}: \mathcal{L}_{\Delta}(\mathcal{T}) \rightarrow \mathcal{L}_{\Delta}(\mathcal{T})$ is given by $[\varphi]^{-1}=\left[\varphi^{-1}\right]$ 
for each $\varphi \in \operatorname{Iso}(\mathcal{T})$. It can be shown that these operations on $\mathcal{L}$ are well defined and that $\mathcal{L}_{\Delta}(\mathcal{T})$ is a locality [Che13, Lemmas A.7,A.9,A.13].

Recall that $T$ denotes the category of transporter systems with isomorphisms and $L$ denotes the category of localities with isomorphisms. We write $T_{0}$ and $L_{0}$ for the categories of transporter systems and localities with rigid isomorphisms.

Theorem 2.11 (cf. Chermak [Che13, Appendix]). The categories $\mathrm{T}$ and $\mathrm{L}$ are equivalent via a functor that restricts to an equivalence between $\mathrm{T}_{0}$ and $\mathrm{L}_{0}$.

Remark 2.12. Strictly speaking, in order for the restriction of the functor $T \rightarrow L$ (to be constructed in the proof) to induce an equivalence between $T_{0}$ and $L_{0}$, we must make two canonical identifications of $S$ with other incarnations of $S$. It is possible that a more precise statement could be made involving a category of $S$-rigid localities, where an $S$-rigid locality is a locality $\mathcal{L}$ together with an embedding $S \hookrightarrow \mathcal{L}$ of partial groups that satisfies natural conditions. But we do not pursue that, because our interest here is mainly in Corollary 2.13.

Proof of Theorem 2.11. Define functors $\Theta: L \rightarrow T$ and $\Lambda: T \rightarrow L$ as follows. On objects, the functors are as described in Subsections 2.3.1 and 2.3.2. Let $\gamma: \mathcal{L} \rightarrow \mathcal{L}^{\prime}$ be an isomorphism between the two localities $(\mathcal{L}, \Delta, S)$ and $\left(\mathcal{L}^{\prime}, \Delta^{\prime}, S^{\prime}\right)$. Define a functor $\Theta(\gamma): \mathcal{T}_{\Delta}(\mathcal{L}) \rightarrow \mathcal{T}_{\Delta^{\prime}}\left(\mathcal{L}^{\prime}\right)$ by the rule

$$
\begin{aligned}
P & \mapsto \gamma(P), \\
(f, P, Q) & \mapsto(\gamma(f), \gamma(P), \gamma(Q)) .
\end{aligned}
$$

$\Theta(\gamma)$ is an invertible functor with inverse $\Theta\left(\gamma^{-1}\right)$, it is clearly isotypical, it sends inclusions to inclusions because $\gamma(1)=1$ and hence it is an isomorphism of transporter systems. Observe that if $\Delta=\Delta^{\prime}$ (so $\left.S=S^{\prime}\right)$ and $\gamma$ is a rigid isomorphism, then $\Theta(\gamma)\left(\delta_{S}(s)\right)=(s, S, S)=\delta_{S}^{\prime}(s)$ for each $s \in S$, so $\Theta(\gamma)$ is a rigid isomorphism of transporter systems. It is then clear that $\Theta$ determines a functor $L \rightarrow T$, which restricts to send $L_{0} \rightarrow T_{0}$.

Conversely, given an isomorphism $\alpha: \mathcal{T} \rightarrow \mathcal{T}^{\prime}$, form the associated localities $\left(\mathcal{L}_{\Delta}(\mathcal{T}), \Delta, S\right)$ and $\left(\mathcal{L}_{\Delta^{\prime}}\left(\mathcal{T}^{\prime}\right), \Delta^{\prime}, S^{\prime}\right)$ and define a function $\Lambda(\alpha): \mathcal{L}_{\Delta}(\mathcal{T}) \rightarrow \mathcal{L}_{\Delta^{\prime}}\left(\mathcal{T}^{\prime}\right)$ via $\Lambda(\alpha)([\varphi])=[\alpha(\varphi)]^{\prime}$, where here we write [-] for equivalence classes in $\operatorname{Iso}\left(\mathcal{T}^{\prime}\right)$. Because $\alpha$ is invertible, it induces a bijection $\Delta \rightarrow \Delta^{\prime}$ sending $S \mapsto S^{\prime}$ and a bijection $\operatorname{Iso}(\mathcal{T}) \rightarrow \operatorname{Iso}\left(\mathcal{T}^{\prime}\right)$. Because $\alpha$ sends inclusions to inclusions, it preserves $\uparrow$ and $\equiv$, and hence $\Lambda(\alpha)$ is a well-defined bijection. Given that $\alpha$ is a functor, it follows from the definition of multiplication in $\mathcal{L}_{\Delta}(\mathcal{T})$ and [Che13, Lemma A.7(b)] that $\Lambda(\alpha)$ is a partial group homomorphism. Then $\Lambda(\alpha)$ restricts to a homomorphism from $S$ to $S^{\prime}$ (if we identify these with $\left\{\left[\delta_{S}(s)\right] \mid s \in S\right\}$ and $\left\{\left[\delta_{S^{\prime}}^{\prime}\left(s^{\prime}\right)\right] \mid s^{\prime} \in S^{\prime}\right\}$ via $\delta$ and $\delta^{\prime}$, respectively), because $\alpha$ is isotypical. Further, if $\alpha$ is rigid, then this translates directly to the condition that $\Lambda(\alpha)$ is a rigid isomorphism of localities. Again, $\Lambda\left(\alpha^{-1}\right)$ is the inverse of $\Lambda(\alpha)$, and so $\Lambda(\alpha)$ is an isomorphism of localities. Thus, $\Lambda$ is a functor that restricts to send $\mathrm{T}_{0} \rightarrow \mathrm{L}_{0}$.

Define $\eta: \mathrm{id}_{\mathrm{T}} \rightarrow \Theta \circ \Lambda$ as follows. For any transporter system $\mathcal{T}, \eta_{\mathcal{T}}: \mathcal{T} \rightarrow \Theta(\Lambda(\mathcal{T}))$ sends each object to itself, and it sends a morphism $\varphi: P \rightarrow Q$ in $\mathcal{T}$ to the triple $\left(\left[\varphi_{0}\right], P, Q\right)$, where $\varphi_{0}$ is the unique morphism from $P$ to $Q_{0}:=\pi(\varphi)(P)$ in $\mathcal{T}$ such that $\delta_{Q_{0}, Q}(1) \circ \varphi_{0}=\varphi$. We will show that $\eta$ is a natural isomorphism of functors. By [Che13, Lemma A.15], $\eta_{\mathcal{T}}$ is a rigid isomorphism of transporter systems, provided that we make the identification of $S$ with the group of equivalence classes $\left\{\left(\left[\delta_{S}(s)\right], S, S\right) \mid s \in S\right\}$ via the canonical isomorphism. Let now $\alpha: \mathcal{T} \rightarrow \mathcal{T}^{\prime}$ be any isomorphism of transporter systems, and consider the naturality diagram

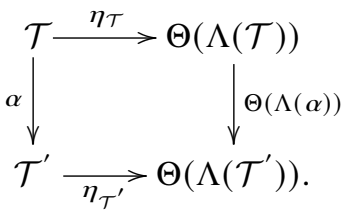


Fix a morphism $\varphi: P \rightarrow Q$ in $\mathcal{T}$. Then

$$
\Theta(\Lambda(\alpha))\left(\left[\varphi_{0}\right], P, Q\right)=\left(\left[\alpha\left(\varphi_{0}\right)\right], \alpha(P), \alpha(Q)\right)
$$

and

$$
\eta_{\mathcal{T}^{\prime}}(\alpha(\varphi))=\left(\left[\alpha(\varphi)_{0}\right], \alpha(P), \alpha(Q)\right)
$$

where $\alpha(\varphi)_{0}$ is the unique morphism from $\alpha(P)$ to $Q_{1}:=\pi^{\prime}(\alpha(\varphi))(\alpha(P))$ such that $\alpha(\varphi)=$ $\delta_{Q_{1}, \alpha(Q)}(1) \circ \alpha(\varphi)_{0}$. Note also that $\alpha(\varphi)=\delta_{\alpha\left(Q_{0}\right), \alpha(Q)}(1) \circ \alpha\left(\varphi_{0}\right)$ as $\alpha$ sends inclusions to inclusions. Thus, to show that $\eta$ is natural, it suffices by uniqueness of restrictions (Lemma 2.2(b)) to show that $Q_{1}=\alpha\left(Q_{0}\right)$. To this end, let $\beta$ be the isomorphism from $S$ to $S^{\prime}$ associated with $\alpha$ in Proposition 2.5. By Proposition 2.5, $\alpha(P)=\beta(P)$ for each $P \in \Delta$, and we have

$$
\pi^{\prime}(\alpha(\varphi))(\alpha(P))=c_{\beta}(\pi(\varphi))(\beta(P))=\beta(\pi(\varphi)(P))=\alpha(\pi(\varphi)(P)),
$$

as required. This completes the proof that $\eta$ is a natural isomorphism.

Next, given a locality $(\mathcal{L}, \Delta, S)$, define $\zeta_{\mathcal{L}}: \mathcal{L} \rightarrow(\Lambda \circ \Theta)(\mathcal{L})$ by

$$
\zeta_{\mathcal{L}}(f)=\left[\left(f, S_{f},{ }^{f} S_{f}\right)\right]
$$

We will show that $\zeta=\left(\zeta_{\mathcal{L}}\right): \mathrm{id}_{\mathrm{L}} \rightarrow \Lambda \circ \Theta$ is a natural isomorphism. Let $\left(f_{n}, \ldots, f_{1}\right) \in \mathbf{D}(\mathcal{L})$, and set $f=\Pi\left(f_{n}, \ldots, f_{1}\right)$. By Definition $2.8(\mathrm{~L} 1 \mathrm{a})$, there are objects $P_{0}, \ldots, P_{n} \in \Delta$ such that $P_{i-1} \leqslant S_{f_{i}}$ and ${ }^{f_{i}} P_{i-1}=P_{i}$ for $i=1, \ldots, n$. Then $\left[\left(f_{i}, S_{f_{i}}, f_{i} S_{f_{i}}\right)\right]=\left[\left(f_{i}, P_{i-1}, P_{i}\right)\right]$ by definition of the equivalence class $[-]$, and this implies that $\zeta_{\mathcal{L}}^{*}\left(f_{n}, \ldots, f_{1}\right):=\left(\zeta_{\mathcal{L}}\left(f_{n}\right), \ldots, \zeta_{\mathcal{L}}\left(f_{1}\right)\right) \in \mathbf{D}(\Lambda(\Theta(\mathcal{L})))$. By definition of the product in $\Lambda(\Theta(\mathcal{L}))$, we have

$$
\Pi\left(\zeta_{\mathcal{L}}^{*}\left(f_{n}, \ldots, f_{1}\right)\right)=\left[\left(\Pi\left(f_{n}, \ldots, f_{1}\right), P_{0}, P_{n}\right)\right]=\left[\left(f, P_{0}, P_{n}\right)\right]=\left[\left(f, S_{f},{ }^{f} S_{f}\right)\right]=\zeta_{\mathcal{L}}\left(\Pi\left(f_{n}, \ldots, f_{1}\right)\right),
$$

so $\zeta_{\mathcal{L}}$ is a partial group homomorphism.

There is an extension of Lemma 3.6 of [Che13] in which $S$ and $S^{\prime}$ (and $\Delta$ and $\Delta^{\prime}$ ) need not be equal and for which Chermak's proof remains valid. This will be used to show that $\zeta_{\mathcal{L}}$ is an isomorphism of localities. The typical element of $\Lambda(\Theta(\mathcal{L}))$ has the form $[(f, P, Q)]$ for $f \in \mathcal{L}, P \leqslant S_{f}$ and $Q \geqslant{ }^{f} P$. It is the image of $f$ under $\zeta_{\mathcal{L}}$, because $\zeta_{\mathcal{L}}(f)=\left[\left(f, S_{f},{ }^{f} S_{f}\right)\right]=[(f, P, Q)]$ by the commutative diagram

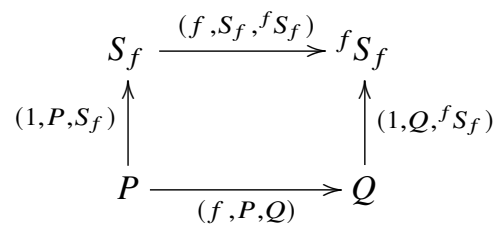

in $\Theta(\mathcal{L})$, so $\zeta_{\mathcal{L}}$ is surjective.

Set $S^{\prime}=\{[(s, S, S)] \mid s \in S\} \leqslant \Lambda(\Theta(\mathcal{L}))$, and fix $s \in S$ and $f \in \mathcal{L}$. Then $\left(f, s, f^{-1}\right) \in \mathbf{D}(\mathcal{L})$ via $X \in \Delta$ if and only if

$$
\left(\left[\left(f,{ }^{s} f^{-1} X,{ }^{f} s f^{-1} X\right)\right],\left[\left(s,{ }^{-1} X,{ }^{s-1} X\right)\right],\left[\left(f^{-1}, X,{ }^{-1} X\right)\right]\right) \in \mathbf{D}(\Lambda(\Theta(\mathcal{L})))
$$

by definition of the domain of the locality built out of the transporter system $\Theta(\mathcal{L})$. Moreover, in this case, $f s f^{-1} \in S$ via $X \in \Delta$ if and only if

$$
\left[\left(f s f^{-1}, X,{ }^{f s f^{-1}} X\right)\right]=\left[\left(f,{ }^{s} f^{-1} X,{ }^{f s f^{-1}} X\right) \circ\left(s,{ }^{-1} X,{ }^{s}{ }^{-1} X\right) \circ\left(f^{-1}, X,{ }^{-1} X\right)\right] \in S^{\prime} .
$$

This shows that $\zeta_{\mathcal{L}}\left(S_{f}\right)=S_{\zeta_{\mathcal{L}}(f)}^{\prime}$. 
Let $h \in \operatorname{ker}\left(\zeta_{\mathcal{L}}\right)$. Then $\left[\left(h, S_{h},{ }^{h} S_{h}\right)\right]=1_{\Lambda(\Theta(\mathcal{L}))}=[(1, S, S)]$. This means that $\left(h, S_{h},{ }^{h} S_{h}\right)$ is a restriction of $(1, S, S)$; that is, $\left(1, S_{h}, S\right)=\left(h, S_{h}, S\right)$, and hence $h=1$. This completes the check of the hypotheses of the extension of [Che13, Lemma 3.6], and so $\zeta_{\mathcal{L}}$ is an isomorphism by that lemma. Moreover, $\zeta_{\mathcal{L}}$ is a rigid isomorphism of localities, provided that we make the identification of $S$ with the group of equivalence classes $\{[(s, S, S)] \mid s \in S\}$ via the canonical isomorphism.

Finally, it remains to verify naturality of $\zeta$. Given another locality $\left(\mathcal{L}^{\prime}, \Delta^{\prime}, S^{\prime}\right)$ and isomorphism $\gamma: \mathcal{L} \rightarrow \mathcal{L}^{\prime}$ mapping $S$ onto $S^{\prime}$, we have for each $f \in \mathcal{L}$ that

$$
\Lambda(\Theta(\gamma))\left(\zeta_{\mathcal{L}}(f)\right)=\left[\left(\gamma(f), \gamma\left(S_{f}\right), \gamma\left({ }^{f} S_{f}\right)\right)\right]^{\prime}
$$

and

$$
\zeta_{\mathcal{L}}(\gamma(f))=\left[\left(\gamma(f), S_{\gamma(f)},{ }^{\gamma(f)} S_{\gamma(f)}\right)\right]^{\prime} .
$$

Because $\gamma$ is an isomorphism mapping $S$ onto $S^{\prime}, \gamma^{*}\left(\mathbf{D}_{\mathcal{L}}(f)\right)=\mathbf{D}_{\mathcal{L}^{\prime}}(\gamma(f))$, and hence $\gamma\left(S_{f}\right)=S_{\gamma(f)}$. In addition, $\gamma\left({ }^{f} P\right)=\gamma(f) \gamma(P)$ for each $P \in \Delta$ and $f \in \mathcal{L}$. This establishes naturality and completes the proof of the theorem.

Corollary 2.13. Fix a transporter system $(\mathcal{T}, \pi, \delta)$ and let $\mathcal{L}_{\Delta}(\mathcal{T})$ be the associated locality. Then the map

$$
\Phi: \operatorname{Aut}(\mathcal{T}) \longrightarrow \operatorname{Aut}\left(\mathcal{L}_{\Delta}(\mathcal{T})\right)
$$

given by sending an automorphism $\alpha \in \operatorname{Aut}(\mathcal{T})$ to the map $\mathcal{L}_{\Delta}(\mathcal{T}) \rightarrow \mathcal{L}_{\Delta}(\mathcal{T})$, which sends a class $[\varphi]$ to $[\alpha(\varphi)]$, for each $\varphi \in \operatorname{Iso}(\mathcal{T})$, is an isomorphism of groups. Moreover, $\Phi$ maps Aut $_{0}(\mathcal{T})$ onto $\operatorname{Aut}_{0}\left(\mathcal{L}_{\Delta}(T)\right)$.

Proof. This follows directly from Theorem 2.11.

Remark 2.14. The obstruction theory for the existence and uniqueness of centric linking systems 'up to isomorphism' as given by Broto, Levi, and Oliver [BLO03, Theorem 3.1] (see also [AKO11, III.5.11]) holds, of course, with respect to the notion of isomorphism of centric linking systems used there. By Proposition 2.5 and Corollary 2.13, this definition coincides with the notion of 'rigid isomorphism' of the associated localities. Thus, Theorem 3.4 of [Oli13] and Theorem 1.1 of [GL16] imply that any two centric linking localities (i.e., $\Delta$-linking systems with $\Delta=\mathcal{F}^{c}$ in the terminology of [Che13, p.49]) associated to a given saturated fusion system are rigidly isomorphic in the sense of [Che13].

\subsection{Linking systems and linking localities}

Theorems 1.1 and 1.2 do not hold for arbitrary localities and transporter systems, as can be seen by considering an appropriate finite group $G$ of the form $O_{p^{\prime}}(G) \times H$, with $O_{p^{\prime}}(G)$ supporting an automorphism of order $p^{2}$ and forming a locality as in the standard Example 2.9.

Definition 2.15. A finite group $N$ is of characteristic $p$ if $C_{N}\left(O_{p}(N)\right) \leqslant O_{p}(N)$. A linking locality is a locality $(\mathcal{L}, \Delta, S)$ such that $\mathcal{F}_{S}(\mathcal{L})$ is saturated, $\mathcal{F}_{S}(\mathcal{L})^{c r} \subseteq \Delta$ and $N_{\mathcal{L}}(P)$ is of characteristic $p$ for each $P \in \Delta$. A linking system is a transporter system $(\mathcal{T}, \delta, \pi)$ associated with a saturated fusion system $\mathcal{F}$ having object set $\Delta$ such that $\mathcal{F}^{c r} \subseteq \Delta$ and $\operatorname{Aut}_{\mathcal{T}}(P)$ is of characteristic $p$ for each $P \in \Delta$.

The assumption that $\mathcal{L}$ is a linking locality (in Theorem 1.1) or a linking system (in Theorem 1.2) is necessary when applying [GL16, Lemma 8.2], which says that a rigid automorphism of a finite group of characteristic $p$ is conjugation by an element of the center of a Sylow $p$-subgroup.

The definition of linking system appearing in Definition 2.15 was given by Henke [Hen19]. It is more general than the usual definition in [AKO11, Definition III.4.1], which forces each object to be $\mathcal{F}$ quasicentric. In Henke's definition, the objects are forced merely to be a subset of the larger collection of $\mathcal{F}$-subcentric subgroups of $S$, namely, the subgroups $P$ of $S$ with the property that $O_{p}\left(N_{\mathcal{F}}(Q)\right)$ is $\mathcal{F}$-centric for each fully $\mathcal{F}$-normalised conjugate $Q$ of $P$. The term 'linking locality' also appears first in 
[Hen19] and refers to the same thing as a 'proper locality' in [Che15]. By [Hen19, Proposition 1], the equivalence between localities and transporter systems given in Theorem 2.11 restricts to an equivalence between linking localities and linking systems.

Examples of linking localities include localities of finite groups of Lie type in characteristic $p$, where, by the Borel-Tits theorem, one may take $\Delta$ to be the set of nonidentity subgroups of a Sylow subgroup. On the other hand, every finite group $G$ gives rise to a linking locality on the set $\Delta$ of $\mathcal{F}_{S}(G)$-subcentric subgroups of a Sylow subgroup $S$, the main theorem of [Hen19].

\section{Rigid outer automorphisms of centric linking systems}

In this section, we prove Theorems 1.1 and 1.2 in the case $\Delta=\mathcal{F}^{c}$ and prove Theorem 1.3. Throughout, we fix a saturated fusion system $\mathcal{F}$ over the finite $p$-group $S$ and a linking locality $(\mathcal{L}, \Delta, S)$ on $\mathcal{F}$.

A version of the Alperin-Goldschmidt fusion theorem for linking localities was proved by Chermak and is needed in the proof of Theorem 1.1. We state a special case of it in a flexible form.

Proposition 3.1. Let $\mathcal{C}$ be any conjugation family for $\mathcal{F}$ and let $g \in \mathcal{L}$. Then there are $Q_{i} \in \mathcal{C} \cap \Delta$ and elements $g_{i} \in N_{\mathcal{L}}\left(Q_{i}\right)$ such that $g=g_{n} \cdots g_{1}$.

Proof. Recall, by definition of a linking locality (proper locality), that $\mathcal{F}^{c r} \subseteq \Delta$. Further, the collection $\mathbf{A}(\mathcal{F})$ defined in [Che16, Notation 3.3] is a subset of $\mathcal{F}^{c r}$ and coincides with the collection of $\mathcal{F}$ essential subgroups [AKO11, Definition I.3.2]. So the assertion is a special case of [Che16, Theorem 3.5], given that the collection of $\mathcal{F}$-essential subgroups is contained in any conjugation family (cf. [AKO11, Proposition I.3.3(b)]).

Proposition 3.1 has the immediate consequence that an automorphism that is the identity on $N_{\mathcal{L}}(Q)$ for each $Q \in \mathcal{C} \cap \Delta$ is the identity automorphism of $\mathcal{L}$. We take the opportunity to prove below a more general statement that generalises Lemma 5.4 of [GL16] to the setting of linking localities. We refer to [Cra11, Definition 7.14] for the definition of a positive characteristic $p$-functor $W$, which we call a conjugacy functor for short. There is a mistake in the proof of [GL16, Lemma 5.4], in which $W(Q)$ is claimed to be well placed, given that $Q$ is. This seems unlikely to be true. It is true that $W(Q)$ is conjugate to a well-placed subgroup, and we give a correct argument in the proof of Lemma 3.2.

Lemma 3.2. Let $\tau$ be an automorphism of $\mathcal{L}$. Fix a conjugacy functor $W$ for $\mathcal{F}$, let $\mathcal{C}$ be the associated conjugation family consisting of those subgroups of $S$ that are well placed with respect to $W$ and set

$$
\mathcal{W}=\{Q \in \mathcal{C} \cap \Delta \mid W(Q)=Q\}
$$

Assume that $W(Q) \in \Delta$ and $W(W(Q))=W(Q)$ whenever $Q \in \Delta$. If $\tau$ is the identity on $N_{\mathcal{L}}(Q)$ for each $Q \in \mathcal{W}$, then $\tau$ is the identity automorphism of $\mathcal{L}$.

Proof. Assume first that $W$ is the identity functor. Then $\mathcal{W}=\mathcal{C} \cap \Delta$. Let $\tau \in \operatorname{Aut}(\mathcal{L})$, and assume that $\tau$ is the identity on $N_{\mathcal{L}}(Q)$ for all $Q \in \mathcal{W}=\mathcal{C} \cap \Delta$. For $g \in \mathcal{L}$, there are $Q_{i} \in \mathcal{C} \cap \Delta$ and $g_{i} \in N_{\mathcal{L}}\left(Q_{i}\right)$ such that $g=g_{n} \cdots g_{1}$ by Proposition 3.1. Then $\tau(g)=\tau\left(g_{n}\right) \cdots \tau\left(g_{1}\right)=g_{n} \cdots g_{1}=g$ by assumption. Thus, $\tau$ is the identity automorphism.

Next, we prove the result for general $W$ satisfying the hypotheses. By the previous case with the identity functor in place of $W$, it suffices to show that $\tau$ is the identity on $N_{\mathcal{L}}(Q)$ for each $Q \in \mathcal{C} \cap \Delta$. Proceed by induction on the index of $Q$ in $S$. Assume first that $Q=S$. Because $S \in \mathcal{C}$ (it is contained in every conjugation family), $W(Q)=W(S) \in \mathcal{C} \cap \Delta$ by assumption on $W$. Hence, because $\left.\tau\right|_{N_{\mathcal{L}}(W(S))}=$ $\operatorname{id}_{N_{\mathcal{L}}(W(S))}$ and $N_{\mathcal{L}}(S) \leqslant N_{\mathcal{L}}(W(S)), \tau$ is the identity on $N_{\mathcal{L}}(Q)$. Fix now $Q<S$ and assume that $\tau$ is the identity on $N_{\mathcal{L}}(R)$ for all $R \in \Delta$ with $|R|>|Q|$. Let $g \in \mathcal{L}$ with ${ }^{g} N_{S}(W(Q)) \leqslant S$ and ${ }^{g} W(Q)$ well-placed by [Cra11, Lemma 7.23]. We claim that $\tau$ fixes $g$. Write $g=g_{n} \cdots g_{1}$ for subgroups $R_{i} \in \mathcal{C} \cap \Delta$ and $g_{i} \in N_{\mathcal{L}}\left(R_{i}\right)$ with $R_{i} \geqslant g_{i} \cdots g_{1} N_{S}(W(Q))$. So $\left|R_{i}\right| \geqslant\left|N_{S}(W(Q))\right| \geqslant\left|N_{S}(Q)\right|>|Q|$. The claim now follows from the inductive hypothesis. Because ${ }^{g} W(Q)$ is well placed and $\Delta$ is closed under 
$\mathcal{L}$-conjugation, we have ${ }^{g} W(Q) \in \mathcal{C} \cap \Delta$. Now $N_{\mathcal{L}}\left({ }^{g} Q\right) \leqslant N_{\mathcal{L}}\left({ }^{g} W(Q)\right)$ by the axioms for a conjugacy functor. Because $\tau$ is the identity on $N_{\mathcal{L}}\left({ }^{g} W(Q)\right)$ by hypothesis, we see that $\tau$ is the identity on $N_{\mathcal{L}}\left({ }^{g} Q\right)$. Finally, because $\tau(g)=g, \tau$ is the identity on $N_{\mathcal{L}}(Q)$, as desired.

Proof of Theorem 1.1 in the case $\Delta=\mathcal{F}^{c}$. Recall that $k(p)=1$ if $p$ is odd, and $k(p)=2$ if $p=2$. Fix $\tau \in \operatorname{Aut}_{0}(\mathcal{L})$. For any finite $p$-group $P$, we take the abelian version of the Thompson subgroup $J(P)$, namely, $J(P)$ is the subgroup generated by the abelian subgroups of $P$ of order $d(P)$, where $d(P)$ is the maximum of the orders of the abelian subgroups of $P$.

We proceed in several steps to complete the proof. The main part of the proof consists in showing that if the automorphism $\tau$ is the identity on $N_{\mathcal{L}}(J(S))$, then $\tau^{k(p)}=\mathrm{id}_{\mathcal{L}}$. This is carried out in Steps 2 to 6 .

Step 1. We first arrange that $\tau$ restricts to the identity automorphism of $N_{\mathcal{L}}(J(S))$. The restriction $\tau$ to $N_{\mathcal{L}}(J(S))$ is an automorphism of $N_{\mathcal{L}}(J(S))$ that is identity on $S \leqslant N_{\mathcal{L}}(J(S))$. Because $\mathcal{L}$ is a linking locality and $J(S) \in \Delta=\mathcal{F}^{c}$, the normaliser $N_{\mathcal{L}}(J(S))$ is of characteristic $p$. Thus, by [GL16, Lemma 8.2], we may fix $z \in Z(S)$ such that $\tau$ is conjugation by $z$ on $N_{\mathcal{L}}(J(S))$. Then upon replacing $\tau$ by $c_{z}^{-1} \tau$, where $c_{z}: \mathcal{L} \rightarrow \mathcal{L}$ denotes the rigid inner automorphism that is (everywhere defined) conjugation by $z$, we complete the proof of Step 1.

Consider the following ordering on $\mathcal{F}^{c}$ :

$$
Q<_{J} P \quad \Longleftrightarrow \quad d(Q)<d(P) \quad \text { or } \quad d(Q)=d(P) \text { and }|J(Q)|<|J(P)| .
$$

We claim that $\tau^{k(p)}$ is the identity on $\mathcal{L}$. Assume the contrary and, using Lemma 3.2 with $W$ the identity functor, choose $Q$ maximal under $<_{J}$ with the property that $N_{\mathcal{L}}(Q)$ is not fixed by $\tau^{k(p)}$.

Step 2. We show that $Q$ may be taken to be well placed with respect to $J$. Let $\mathcal{C}$ be the collection of subgroups of $S$ that are well placed with respect to the Thompson subgroup functor $J$. Then $\mathcal{C}$ forms a conjugation family for $\mathcal{F}$ by [Cra11, Corollary 7.26]. Let $g \in N_{\mathcal{L}}(Q)$ not fixed by $\tau^{k(p)}$. By Proposition 3.1, we may write $g$ as a product of elements $g_{i} \in N_{\mathcal{L}}\left(R_{i}\right)$ with $R_{i} \in \mathcal{C} \cap \Delta$ and where $Q=Q_{0}=Q_{n}, Q_{i}={ }^{g_{i}} Q_{i-1}$ and $R_{i} \geqslant\left\langle Q_{i-1}, Q_{i}\right\rangle$ for each $i$. Because $g$ is not fixed by $\tau^{k(p)}$, some $g_{i}$ is not fixed by $\tau^{k(p)}$. Now because $Q$ is isomorphic to a subgroup of $R_{i}$, we see that $d(Q) \leqslant d\left(R_{i}\right)$. Therefore, equality holds by maximality of $Q$ under $<_{J}$. Then $|J(Q)| \leqslant\left|J\left(R_{i}\right)\right|$, so again equality holds by maximality of $Q$. Hence, upon replacing $Q$ by $R_{i}$, we may assume that $Q \in \mathcal{C}$.

Step 3. Set $H=N_{\mathcal{L}}(Q)$ and $T=N_{S}(Q)$. We next show that $J(Q)=J(Q J(T))$. Because $Q \in \Delta, H$ is of characteristic $p$. By [GL16, Lemma 8.2], we may fix $z \in Z(T)$ such that $\tau$ is conjugation by $z$ on $H$. Then $\tau^{2}$ is conjugation by $z^{2}$ on $H$. Because $\tau^{k(p)}$ is not the identity on $H$, we have that $z^{k(p)}$ is not centralised by $H$. Applying [Gla68, Theorem A], we conclude that $z^{k(p)}$ is not centralised by $N_{H}(J(T))$. Now $N_{H}(J(T)) \leqslant N_{H}(Q J(T))$ because $H=N_{H}(Q)$, so that $\tau^{k(p)}$ is not the identity on $N_{\mathcal{L}}(Q J(T))$. Because $Q J(T) \in \mathcal{F}^{c}$ and $d(Q) \leqslant d(Q J(T))$, we have equality by maximality of $Q$ under $<_{J}$. Then $J(Q) \leqslant J(Q J(T))$, and so

$$
J(Q)=J(Q J(T))
$$

again by maximality of $Q$ under $<_{J}$.

Step 4. Here we show $J(T)=J(Q)$. Because $d(Q) \leqslant d(T)=d(J(T)) \leqslant d(Q J(T))$, we have equality by Step 3. Thus, $d(Q)=d(T)$ and $Q \leqslant T$ yield that $J(Q) \leqslant J(T) \leqslant J(Q J(T))$, and again we have equality by choice of $Q$. This completes the proof of Step 4 .

Step 5. We next show that $J(Q)$ is $\mathcal{F}$-centric. Suppose on the contrary that $J(Q)$ is not $\mathcal{F}$-centric. By Step 2, $Q$ is well placed. By definition of well placed, $J(T)$ is fully $\mathcal{F}$-normalised. Hence, $J(Q)$ is fully $\mathcal{F}$-normalised by Step 4 . Because $J(Q)$ is fully $\mathcal{F}$-normalised and not $\mathcal{F}$-centric, we have $C_{S}(J(Q)) \not J J(Q)$. Note that $C_{S}(J(Q)) \not \leq Q$ because $J(Q)$ does contain its centraliser in $Q$. Hence, $Q C_{S}(J(Q))>Q$, so with $R:=N_{Q C_{S}(J(Q))}(Q)$, we have

$$
R>Q \text {. }
$$


On the other hand, Step 4 shows that

$$
R=Q N_{C_{S}(J(Q))}(Q)=Q C_{T}(J(Q))=Q C_{T}(J(T))=Q Z(J(T))=Q Z(J(Q))=Q,
$$

a contradiction.

Step 6. Lastly, we obtain a contradiction. Among all well-placed, $\mathcal{F}$-centric subgroups maximal under $<_{J}$ whose normaliser in $\mathcal{L}$ is not centralised by $\tau^{k(p)}$, choose $Q$ of minimum order. By Step 4 and the definition of well placed, $J(Q)=J(T)$ is well placed. By Step 5, $J(Q)$ is centric. Note that $\tau^{k(p)}$ is not the identity on $N_{H}(J(Q))=H$ by choice of $Q$. Because $d(Q)=d(J(Q))$ and $J(J(Q))=J(Q)$, we have that $Q=J(Q)$ by minimality of $|Q|$. Therefore, by Step 4,

$$
J(Q)=J(T)=J\left(N_{S}(Q)\right)=J\left(N_{S}(J(Q))\right) .
$$

It now follows that $Q=J(Q)=J(S)$ by [GL16, Lemma 8.5(b)]. Because $N_{\mathcal{L}}(J(S))$ is centralised by $\tau$ by Step 1, this is a contradiction.

Step 7. We prove the splitting condition. Because Steps 1 to 6 show that $\operatorname{Out}_{0}(\mathcal{L})=1$ if $p$ is odd, splitting is trivial in that case. So take $p=2$. Let $E$ be the subgroup of $\operatorname{Aut}_{0}(\mathcal{L})$ consisting of those automorphisms that restrict to the identity on $N_{\mathcal{L}}(J(S))$. Step 1 shows that $E$ maps surjectively onto $\operatorname{Out}_{0}(\mathcal{L})$ via the quotient map $\operatorname{Aut}_{0}(\mathcal{L}) \rightarrow \operatorname{Out}_{0}(\mathcal{L})$, and Steps 1 to 6 show that $E$ is a vector space over $\mathbb{F}_{2}$. There is therefore a subgroup $E_{0}$ that is a complement to $C_{\mathrm{Aut}_{\mathcal{Z}(S)}(\mathcal{L})}\left(N_{\mathcal{L}}(J(S))\right)$ in $E$ and that maps isomorphically onto $\operatorname{Out}_{0}(\mathcal{L})$. This proves the assertion.

Proof of Theorem 1.2 when $\mathcal{L}$ is a centric linking system. This follows directly from Theorem 1.1 in the centric linking locality case, given Theorem 2.11 .

Remark 3.4. The method of proof of Theorems 1.1 and 1.2 in case $\Delta=\mathcal{F}^{c}$ shows the slightly stronger conclusion: If $\tau$ is an automorphism of a centric linking locality (centric linking system) that is the identity on $N_{\mathcal{L}}(J(S))\left(\operatorname{Aut}_{\mathcal{L}}(J(S))\right.$, then $\tau^{k(p)}=\operatorname{id}_{\mathcal{L}}$.

We next want to prove Theorem 1.3, but first recall certain definitions from [AKO11, Section III.5]. Let $\mathcal{O}\left(\mathcal{F}^{c}\right)$ be the category with objects the $\mathcal{F}$-centric subgroups and with morphism sets

$$
\operatorname{Mor}_{\mathcal{O}\left(\mathcal{F}^{c}\right)}(P, Q)=\operatorname{Inn}(Q) \backslash \operatorname{Hom}_{\mathcal{F}}(P, Q),
$$

the set of orbits of $\operatorname{Inn}(Q)$ in its left action by composition. The center functor

$$
\mathcal{Z}_{\mathcal{F}}: \mathcal{O}\left(\mathcal{F}^{c}\right) \rightarrow A b
$$

is the functor that sends a subgroup $P$ to its center $Z(P)$ and sends a morphism $[\varphi]: P \rightarrow Q$ to the composite $Z(Q) \hookrightarrow Z(\varphi(P)) \stackrel{\varphi^{-1} I_{Z(\varphi(P))}}{\longrightarrow} Z(P)$ induced by the restriction of $\varphi^{-1}: \varphi(P) \rightarrow P$ to $Z(\varphi(P))$.

We refer to Subsection III.5.1 of [AKO11] for a description of the bar resolution for functor cohomology and write $d$ for the coboundary map. Recall that a 0 -cochain for $\mathcal{Z}_{\mathcal{F}}$ sends an object $P$ of $\mathcal{O}\left(\mathcal{F}^{c}\right)$ to an element in $Z(P)$. A 1 -cochain sends a morphism $P \stackrel{[\varphi]}{\longrightarrow} Q$ in the orbit category to an element in $Z(P)$. A 1-cochain for $\mathcal{Z}_{\mathcal{F}}$ is said to be inclusion-normalised if it sends the class of each inclusion $\iota_{P}^{Q}$ to $1 \in Z(P)$. Write $\widehat{Z}^{1}\left(\mathcal{O}\left(\mathcal{F}^{c}\right), \mathcal{Z}_{\mathcal{F}}\right)$ for the group of inclusion-normalised 1-cocycles and write $\widehat{B}^{1}\left(\mathcal{O}\left(\mathcal{F}^{c}\right), \mathcal{Z}_{\mathcal{F}}\right) \subseteq \widehat{Z}^{1}\left(\mathcal{O}\left(\mathcal{F}^{c}\right), \mathcal{Z}_{\mathcal{F}}\right)$ for the group of inclusion-normalised 1-coboundaries.

By the proof of [AKO11, III.5.12], there is a group homomorphism

$$
\tilde{\lambda}: \widehat{Z}^{1}\left(\mathcal{O}\left(\mathcal{F}^{c}\right), \mathcal{Z}_{\mathcal{F}}\right) \rightarrow \operatorname{Aut}(\mathcal{L})
$$


given by sending a 1-cocycle $t$ to the automorphism of $\mathcal{L}$ that is the identity on objects and that sends a morphism $\varphi: P \rightarrow Q$ in $\mathcal{L}$ to $\varphi \circ \delta_{P}(t([\varphi]))$. Next, consider the group homomorphisms

$$
\text { cnst: } Z(S) \rightarrow C^{0}\left(\mathcal{O}\left(\mathcal{F}^{c}\right), \mathcal{Z}_{\mathcal{F}}\right) \quad \text { and } \quad \text { conj: } Z(S) \rightarrow \operatorname{Aut}_{0}(\mathcal{L}),
$$

where cnst sends an element $z \in Z(S)$ to the constant 0-cochain $u_{z}$ with value $z$ on each centric subgroup, and conj sends an element $z$ to the conjugation automorphism $c_{\delta_{S}(z)} \in \operatorname{Aut}_{0}(\mathcal{L})$.

Lemma 3.5. There is an isomorphism of short exact sequences

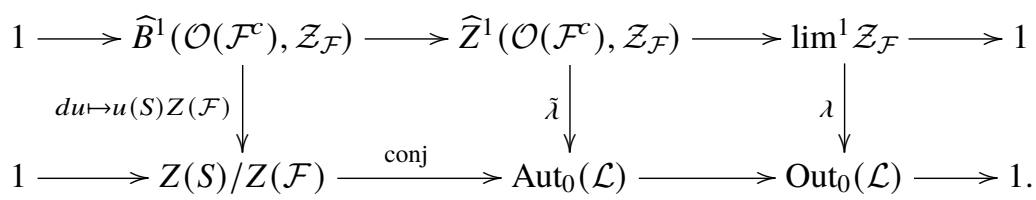

Proof. This is essentially contained in the proof of [AKO11, Proposition III.5.12]. There the groups $\operatorname{Aut}(\mathcal{L})$ and $\operatorname{Out}(\mathcal{L})$ are denoted $\operatorname{Aut}_{\text {typ }}^{I}(\mathcal{L})$ and $\operatorname{Out}_{\text {typ }}(\mathcal{L})$. The commutative diagram displayed on [AKO11, p.186] is shown to have exact rows and columns. Thus, $\tilde{\lambda}: \widehat{Z}^{1}\left(\mathcal{O}\left(\mathcal{F}^{c}\right), \mathcal{Z}_{\mathcal{F}}\right) \rightarrow \operatorname{Aut}(\mathcal{L})$ is injective with image $\operatorname{ker}(\tilde{\mu})=\operatorname{Aut}_{0}(\mathcal{L})$. In addition, $\tilde{\lambda}$ induces an injective homomorphism $\lambda: \lim ^{1} \mathcal{Z}_{\mathcal{F}} \rightarrow \operatorname{Out}(\mathcal{L})$ with image $\operatorname{ker}(\mu)=\operatorname{Out}_{0}(\mathcal{L})$, and so $\tilde{\lambda}$ and $\lambda$ are isomorphisms after restricting to these codomains. Thus, the commutativity of this diagram also gives that the right square in (3.6) commutes.

Second, from the proof of [AKO11, III.5.12], the composite $d$ ocnst has image $\widehat{B}^{1}\left(\mathcal{O}\left(\mathcal{F}^{c}\right), \mathcal{Z}_{\mathcal{F}}\right)$, where, for each $z \in Z(S)$, the image $d u_{z}$ of $u_{z}$ under the coboundary map is inclusion normalised, and $\tilde{\lambda}\left(d u_{z}\right)$ is conjugation by $\delta_{S}(z)$ on $\mathcal{L}$. The composite $\widehat{B}^{1}\left(\mathcal{O}\left(\mathcal{F}^{c}\right), \mathcal{Z}_{\mathcal{F}}\right) \hookrightarrow \widehat{Z}^{1}\left(\mathcal{O}\left(\mathcal{F}^{c}\right), \mathcal{Z}_{\mathcal{F}}\right) \stackrel{\tilde{\lambda}}{\rightarrow} \operatorname{Aut}_{0}(\mathcal{L})$ is injective. Thus, the kernel of the composite $d \circ$ cnst is the same as the kernel of conj. But $\operatorname{ker}(\operatorname{conj})=Z(\mathcal{F})$ by [AOV12, Lemma 1.14]. Therefore, the inverse $d u \mapsto u(S) Z(\mathcal{F})$ of the isomorphism $Z(S) / Z(\mathcal{F}) \rightarrow$ $\widehat{B}^{1}\left(\mathcal{O}\left(\mathcal{F}^{c}\right), \mathcal{Z}_{\mathcal{F}}\right)$ induced by $d \circ$ cnst makes the left square in (3.6) commute.

Proof of Theorem 1.3. By Theorem 1.2 in the case $\Delta=\mathcal{F}^{c}$, the sequence $1 \rightarrow \operatorname{Aut}_{Z(S)}(\mathcal{L}) \rightarrow$ $\operatorname{Aut}_{0}(\mathcal{L}) \rightarrow \operatorname{Out}_{0}(\mathcal{L}) \rightarrow 1$ is split exact. Because $\operatorname{Aut}_{Z(S)}(\mathcal{L})$ is the image of the conjugation map $Z(S) / Z(\mathcal{F}) \rightarrow \operatorname{Aut}_{0}(\mathcal{L})$, it follows from Lemma 3.5 that the sequence $1 \rightarrow \widehat{B}^{1}\left(\mathcal{O}\left(\mathcal{F}^{c}\right), \mathcal{Z}_{\mathcal{F}}\right) \rightarrow$ $\widehat{Z}^{1}\left(\mathcal{O}\left(\mathcal{F}^{c}\right), \mathcal{Z}_{\mathcal{F}}\right) \rightarrow \lim ^{1} \mathcal{Z}_{\mathcal{F}} \rightarrow 1$ is also split exact and that $\lim ^{1} \mathcal{Z}_{\mathcal{F}} \cong \operatorname{Out}_{0}(\mathcal{L})$ is elementary abelian.

\section{Extending to larger object sets}

In this section, we observe via Chermak descent [Che13, Theorem 5.15] that the group of rigid automorphisms does not change when a centric linking locality is expanded to a larger object set. Recall from [Hen19] that a subgroup $P$ of $S$ is said to be $\mathcal{F}$-subcentric if for each fully $\mathcal{F}$-normalised $\mathcal{F}$-conjugate $Q$ of $P$ the subgroup $O_{p}\left(N_{\mathcal{F}}(Q)\right)$ is $\mathcal{F}$-centric. The set of $\mathcal{F}$-subcentric subgroups is denoted $\mathcal{F}^{s}$.

Proposition 4.1. Let $\mathcal{L}^{+}$be a linking locality with object set $\Delta^{+}$and fusion system $\mathcal{F}$ over a p-group $S$. Let $\Delta \subseteq \Delta^{+}$be a subset that contains $\mathcal{F}^{c r}$ and is closed under $\mathcal{F}$-conjugacy and passing to overgroups. Assume that $\left.\mathcal{L}^{+}\right|_{\Delta}=\mathcal{L}$. Then restriction induces an isomorphism $\operatorname{Aut}_{0}\left(\mathcal{L}^{+}\right) \rightarrow \operatorname{Aut}_{0}(\mathcal{L})$ that restricts to an isomorphism $\operatorname{Aut}_{Z(S)}\left(\mathcal{L}^{+}\right) \rightarrow \operatorname{Aut}_{Z(S)}(\mathcal{L})$.

Proof. This follows from Corollary 5.16 of [Che13], applied in the same way as in [Hen19, Theorem 7.2]. The proof is by induction on $\left|\Delta^{+}-\Delta\right|$. If $\Delta^{+}=\Delta$, then $\mathcal{L}^{+}=\mathcal{L}$ and there is nothing to prove. Let $T \in \Delta^{+}-\Delta$ be maximal under inclusion. We claim that Hypothesis 5.3 of [Che13] holds. Because $\Delta$ and $\Delta^{+}$are $\mathcal{F}$-invariant and closed under passing to overgroups, we can replace $T$ by an $\mathcal{F}$-conjugate if necessary and assume that $T$ is fully $\mathcal{F}$-normalised. By induction, we may also assume that $\Delta^{+}=\Delta \cup T^{\mathcal{F}}$. 
Let $\widehat{T}=O_{p}\left(N_{\mathcal{F}}(T)\right)$. Then $T \leqslant \widehat{T}$, and we claim that the inclusion is proper. Assume otherwise. As an object of a linking locality, $T$ is $\mathcal{F}$-subcentric by [Hen19, Proposition 1(b)]. So by [Hen19, Proposition 3.18], it follows that $T \in \mathcal{F}^{c r}$. But then $T \in \Delta$, which contradicts the choice of $T$. Thus, $T<\widehat{T}$, so $\widehat{T} \in \Delta$ by choice of $T$.

Let $M=N_{\mathcal{L}}(T)$, and set

$$
\Delta_{T}:=\left\{N_{P}(T) \mid T \leqslant P \in \Delta\right\}=\left\{P \in \Delta \mid T \leqslant P \leqslant N_{S}(T)\right\},
$$

where the second equality comes from maximality of $T$ in $\Delta^{+}-\Delta$. By Lemma 7.1 of [Hen19], $M$ is a finite group that is a model for $N_{\mathcal{F}}(T)$. In particular, $T$ is normal in $M$ and $N_{S}(T)$ is a Sylow $p$-subgroup of $M$. So, indeed, taking the identity $\mathcal{L} \rightarrow \mathcal{L}$ as a rigid automorphism, Hypothesis 5.3 of [Che13] holds. Recall the locality $\mathcal{L}_{\Delta_{T}}(M)$ from Example 2.9, and note that $\mathcal{L}_{\Delta_{T}}(M)=M$ in the current situation, because each normal $p$-subgroup of the fusion system of $M$ is normal in $M$ [Hen19, Theorem 2.1(b)]. By Corollary 5.16 of [Che13], there is a unique rigid isomorphism $\mathcal{L}^{+}\left(\mathrm{id}_{M}\right) \rightarrow \mathcal{L}^{+}$that restricts to the identity on $\mathcal{L}$, where the former is constructed in [Che13, Theorem 5.14] and defined after the proof of [Che13, Theorem 5.14]. Identify $\mathcal{L}^{+}\left(\mathrm{id}_{M}\right)$ and $\mathcal{L}^{+}$via this isomorphism. The identity automorphism is then the unique rigid automorphism of $\mathcal{L}^{+}$that is the identity on $\mathcal{L}$. This shows that the restriction map $\operatorname{Aut}_{0}\left(\mathcal{L}^{+}\right) \rightarrow \operatorname{Aut}_{0}(\mathcal{L})$ is injective.

To see surjectivity of restriction, take an arbitrary rigid isomorphism $\beta$ of $\mathcal{L}$. Again by [Che13, Corollary 5.16], there is a rigid isomorphism $\beta^{+}: \mathcal{L}^{+}\left(\left.\beta\right|_{M}\right) \rightarrow \mathcal{L}^{+}$that restricts to $\beta$ on $\mathcal{L}$. Taking now $\mathcal{L}^{+}\left(\beta_{M}\right)$ in the role of $\mathcal{L}^{+}$, we see that there is also a rigid isomorphism id $\mathrm{id}^{+} \mathcal{L}^{+}=\mathcal{L}^{+}\left(\mathrm{id}_{M}\right) \rightarrow \mathcal{L}^{+}\left(\beta_{M}\right)$ that is the identity on $\mathcal{L}$. The composition $\beta^{+} \circ \mathrm{id}^{+} \in \operatorname{Aut}_{0}\left(\mathcal{L}^{+}\right)$restricts to $\beta$ on $\mathcal{L}$, and this shows that the restriction map is surjective.

Proof of Theorems 1.1 and 1.2. Let $(\mathcal{L}, \Delta, S)$ be an arbitrary linking locality. Now $\Delta \subseteq \mathcal{F}^{s}$ by Proposition 1(b) of [Hen19], so by Theorem 7.2 of [Hen19] there is a linking locality $\left(\mathcal{L}^{+}, \mathcal{F}^{s}, S\right)$ that restricts to $\mathcal{L}$ on $\Delta$. Because $\mathcal{F}^{c} \subseteq \mathcal{F}^{s}$, two applications of Proposition 4.1 give an isomorphism of short exact sequences between $1 \rightarrow \operatorname{Aut}_{Z(S)}(\mathcal{L}) \rightarrow \operatorname{Aut}_{0}(\mathcal{L}) \rightarrow \operatorname{Out}_{0}(\mathcal{L}) \rightarrow 1$ and $1 \rightarrow \operatorname{Aut}_{Z(S)}\left(\left.\mathcal{L}^{+}\right|_{\mathcal{F}^{c}}\right) \rightarrow$ $\operatorname{Aut}_{0}\left(\left.\mathcal{L}^{+}\right|_{\mathcal{F}^{c}}\right) \rightarrow \operatorname{Out}_{0}\left(\left.\mathcal{L}^{+}\right|_{\mathcal{F}^{c}}\right) \rightarrow 1$. Theorem 1.1 now follows from the proof in the case $\Delta=\mathcal{F}^{c}$. Then Theorem 1.2 follows from Theorem 1.1 and Theorem 2.11.

Remark 4.2. Given the results of this section, the stronger statement mentioned in Remark 3.4 applies verbatim to arbitrary linking localities (linking systems) with object set $\Delta$ containing $J(S)$.

\section{Comparing automorphisms of groups and linking systems}

One may wonder whether it is possible to recover from Theorem 1.2 the analogous theorems about groups, namely, [Gla68, Theorem 10] for $p=2$ and [GGLN20, Theorem 3.3] for $p$ odd. This is possible, but the only way we know how to do it goes through an argument similar to existing arguments for establishing the group case anyway, so our way seems to have little additional value. However, in the process of trying to construct a proof, we obtained Theorem 5.1, which appears to be new and of independent interest. It depends for its proof on the $Z_{p}^{*}$-theorem [Gla66a], [GLS98, Proposition 7.8.2 and Remark 7.8.3] that in a finite group with no normal $p^{\prime}$-subgroups, any element that is weakly closed in a Sylow $p$-subgroup is central.

First we need to set up some notation. Let $p$ be a prime and let $G$ be a finite group with Sylow $p$ subgroup $S$. We write $\mathcal{L}=\mathcal{L}_{S}^{c}(G)$ and $\mathcal{F}=\mathcal{F}_{S}(G)$ for the centric linking system and fusion system of $G$. Thus, $\mathcal{L}$ has objects the $\mathcal{F}$-centric subgroups or, equivalently, the $p$-centric subgroups of $G$; that is, the subgroups $P$ of $S$ with $C_{G}(P)=Z(P) \times O_{p^{\prime}}\left(C_{G}(P)\right)$. Morphisms are given by

$$
\operatorname{Mor}_{\mathcal{L}}(P, Q)=N_{G}(P, Q) / O_{p^{\prime}}\left(C_{G}(P)\right),
$$

where $N_{G}(P, Q)=\left\{g \in G \mid{ }^{g} P \leqslant Q\right\}$ is the transporter set, where composition is induced by multiplication in $G$ and where $O_{p^{\prime}}\left(C_{G}(P)\right)$ acts on $N_{G}(P, Q)$ from the right. The structural functor $\delta$ is the inclusion map, and $\pi$ sends a coset $g O_{p^{\prime}}\left(C_{G}(P)\right)$ to conjugation by $g$. 
By Sylow's theorem, each outer automorphism of $G$ is represented by an automorphism $\alpha \in$ $N_{\text {Aut }(G)}(S)$. Such an automorphism induces an isomorphism from $O_{p^{\prime}}\left(C_{G}(P)\right)$ to $O_{p^{\prime}}\left(C_{G}(\alpha(P))\right)$ and a bijection $N_{G}(P, Q) \rightarrow N_{G}(\alpha(P), \alpha(Q))$ for each pair of centric subgroups $P$ and $Q$. It is then straightforward to check that $\alpha$ induces an automorphism of $\mathcal{L}$ by restriction in this way. Let

$$
\tilde{\kappa}_{G}: N_{\operatorname{Aut}(G)}(S) \rightarrow \operatorname{Aut}(\mathcal{L})
$$

denote the resulting group homomorphism. This map sends $\operatorname{Aut}_{G}(S)$ onto $\left\{c_{\gamma} \mid \gamma \in \operatorname{Aut}_{\mathcal{L}}(S)\right\}$, and so there is an induced homomorphism

$$
\kappa_{G}: \operatorname{Out}(G) \rightarrow \operatorname{Out}(\mathcal{L})
$$

The composition $\tilde{\mu}_{G} \circ \tilde{\kappa}_{G}: N_{\operatorname{Aut}(G)}(S) \rightarrow \operatorname{Aut}\left(\mathcal{F}_{S}(G)\right)$ is just restriction to $S$. Here $\tilde{\mu}_{G}$ is defined just after Proposition 2.5.

Theorem 5.1. Fix a prime $p$, a finite group $G$ and a Sylow p-subgroup $S$ of $G$. Let $\mathcal{L}$ be the centric linking system for $G$. If $O_{p^{\prime}}(G)=1$, then $\operatorname{ker}\left(\kappa_{G}\right)$ is a $p^{\prime}$-group.

The proof uses the $Z_{p}^{*}$-theorem only in the semidirect product of $G$ by a $p$-power automorphism. So if $p=2$ or the composition factors of $G$ are known, then this does not depend on the CFSG.

Proof. Assume $O_{p^{\prime}}(G)=1$. Fix $a \in N_{\text {Aut }(G)}(S)$ with $[a] \in \operatorname{ker}\left(\kappa_{G}\right)$ and recall that $\tilde{\mu}_{G} \circ \tilde{\kappa}_{G}$ sends $a$ to $\left.a\right|_{S}$. Because $\tilde{\kappa}_{G}$ maps $N_{\operatorname{Inn}(G)}(S)$ onto $\operatorname{Inn}(\mathcal{L})=\left\{c_{\gamma} \mid \gamma \in \operatorname{Aut}_{\mathcal{L}}(S)\right\}$, we may adjust $a$ by an element of $N_{\operatorname{Inn}(G)}(S)$ and take $a \in C_{\text {Aut }(G)}(S)$. Then by choice of $a, \tilde{\kappa}_{G}(a) \in \operatorname{Inn}(\mathcal{L}) \cap \operatorname{ker}\left(\tilde{\mu}_{G}\right)=\operatorname{Aut}_{Z(S)}(\mathcal{L})$. Choose $z \in Z(S)$ such that $\tilde{\kappa}_{G}(a)=c_{z}$. Replacing $a$ by $a c_{z^{-1}}$, we may take $a \in \operatorname{ker}\left(\tilde{\kappa}_{G}\right)$. Finally, replacing $a$ by a $p^{\prime}$-power, we may take $a$ of $p$-power order.

We will show that, if $[a] \neq 1$ in $\operatorname{Out}(G)$, then $a$ normalises but does not centralise $H / O_{p^{\prime}}(H)$ for some $p$-local subgroup $H=N_{G}(Q)$ with $Q p$-centric in $G$; that is, with $Q \in \mathcal{F}_{S}(G)^{c}$. Thus, $\tilde{\kappa}_{G}(a)$ does not centralise $\operatorname{Aut}_{\mathcal{L}}(Q)$, and hence $\tilde{\kappa}_{G}(a) \neq 1$, contrary to our choice of $a$.

So assume $[a] \neq 1$. Let $\widehat{G}=G\langle a\rangle$ be the semidirect product, and set $\widehat{S}=S\langle a\rangle$. Then $\widehat{S}$ is Sylow in $\widehat{G}$, and $\langle a\rangle \leqslant Z(\widehat{S})$. In addition, $\widehat{S}=S \times\langle a\rangle$ and $Z(\widehat{S})=Z(S) \times\langle a\rangle$. Note that if $a$ is weakly closed in $\widehat{S}$ with respect to $\widehat{G}$, then by the $Z_{p}^{*}$-theorem we have $a \in Z(\widehat{G})$ because $O_{p^{\prime}}(\widehat{G})=O_{p^{\prime}}(G)=1$, so that $a=1$, contrary to assumption.

So $a$ is not weakly closed in $\widehat{S}$ with respect to $\widehat{G}$. By the Alperin-Goldschmidt fusion theorem in $\widehat{G}$, there is a $\mathcal{F}_{\widehat{S}}(\widehat{G})$-centric radical subgroup $\widehat{Q} \leqslant \widehat{S}$ and $\widehat{h} \in N_{\widehat{G}}(\widehat{Q})$ such that $a \in Z(\widehat{S}) \leqslant Z(\widehat{Q})$ and $a \neq \widehat{h}^{\widehat{n}} a \in Z(\widehat{Q})$. By [LO02, Proposition A.11(c)],

$$
Q:=\widehat{Q} \cap G \text { is } \mathcal{F}_{S}(G) \text {-centric radical. }
$$

Write $\widehat{h}=h a^{k}$ for some integer $k$ and some $h \in G$. Because $a^{k} \in \widehat{Q}$ and $Q=\widehat{Q} \cap G$, we have $h \in N_{G}(\widehat{Q}) \leqslant N_{G}(Q)$. In addition, $a \neq \widehat{h}_{a}={ }^{h} a$. So $[a, h] \in \widehat{S}$. Note that $a$ normalises $N_{G}(Q)$, so $a$ normalises $O_{p^{\prime}}\left(N_{G}(Q)\right)$. If $a$ centralises $h$ modulo $O_{p^{\prime}}\left(N_{G}(Q)\right)$, then we would have $[a, h] \in O_{p^{\prime}}\left(N_{G}(Q)\right) \cap \widehat{S}=1$, a contradiction. Hence, $a$ does not centralise $N_{G}(Q) / O_{p^{\prime}}\left(N_{G}(Q)\right)$. Together with (5.2), this completes the proof of the proposition.

A saturated fusion system $\mathcal{F}$ over $S$ is said to be tame if $\mathcal{F}=\mathcal{F}_{S}(G)$ for some finite group $G$ with Sylow $p$-subgroup $S$ such that the map $\kappa_{G}$ is split surjective. Theorem 5.1 can be combined with the following lemma of Broto, Møller, and Oliver to show that the splitting condition in the definition of tame is unnecessary. The version we give of this lemma is a little different from the corresponding statement in [BMO19, Lemma 1.5(b)]: Two occurrences of $O_{p^{\prime}}(Z(G))$ appearing there (in the statement and proof) have been replaced by $O_{p^{\prime}}(G)$. This change helps to make clearer the step in the proof of [BMO19, Lemma 1.5(b)] that reduces to the case in which $Z(G)$ is a $p$-group. The proof of the lemma is otherwise the same. 
Lemma 5.3. Let $G$ be a finite group, $p$ a prime and $S$ a Sylow $p$-subgroup of $G$. Assume that $\kappa_{G}$ is surjective and $\operatorname{ker}\left(\kappa_{G}\right)$ is a $p^{\prime}$-group. Then there is $\widehat{G} \geqslant G / O_{p^{\prime}}(G)$ such that $\kappa_{\widehat{G}}$ is split surjective and such that $\mathcal{F}_{S}(\widehat{G})=\mathcal{F}_{S}(G)$. In particular, $\mathcal{F}_{S}(G)$ is tame, and it is tamely realised by $\widehat{G}$.

Proposition 5.4. Let $\mathcal{F}$ be a saturated fusion system over the p-group $S$. If $\mathcal{F} \cong \mathcal{F}_{S}(G)$ for some finite group $G$ such that the map $\kappa_{G}$ is surjective, then $\mathcal{F}$ is tame. Moreover, there is an extension $\widehat{G} \geqslant G / O_{p^{\prime}}(G)$ of $G / O_{p^{\prime}}(G)$ that tamely realises $\mathcal{F}$.

Proof. Fix such a $G$, let $\bar{G}=G / O_{p^{\prime}}(G)$ and identify $S$ also with its image in $\bar{G}$. Write $\mathcal{F}=\mathcal{F}_{S}(G)$, $\overline{\mathcal{F}}=\mathcal{F}_{S}(\bar{G}), \mathcal{L}=\mathcal{L}_{S}^{c}(G)$ and $\overline{\mathcal{L}}=\mathcal{L}_{S}^{c}(\bar{G})$. The canonical homomorphism $G \rightarrow \bar{G}$ induces isomorphisms $\mathcal{L} \rightarrow \overline{\mathcal{L}}$ and $\mathcal{F} \rightarrow \overrightarrow{\mathcal{F}}$. As in the proof of [AOV12, Lemma 2.19], there is a resulting commutative diagram

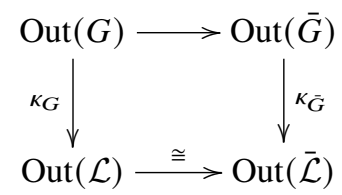

Because $\kappa_{G}$ is surjective, $\kappa_{\bar{G}}$ is also surjective, so we may replace $G$ by $\bar{G}$ and take $O_{p^{\prime}}(G)=1$. The result now follows from Theorem 5.1 and Lemma 5.3.

In [Gla66b], the first author showed, for a core-free group $G$ with Sylow 2-subgroup $S$, that the group $C_{\operatorname{Aut}(G)}(S)$ has abelian 2-subgroups and a normal 2-complement. The following proposition gives further information and a reinterpretation of that situation.

Proposition 5.5. Let $G$ be a finite group with Sylow p-subgroup $S$, let $\mathcal{L}$ be the centric linking system for $G$ and set $A=C_{\operatorname{Aut}(G)}(S) / C_{\operatorname{Inn}(G)}(S)$. If $O_{p^{\prime}}(G)=1$, then $A \cong O_{p^{\prime}}(A) \rtimes B$ where $B=1$ if $p$ is odd and where $B$ is an elementary abelian 2-group if $p=2$. The normal $p$-complement $O_{p^{\prime}}(A)$ is the subgroup of $N_{\operatorname{Aut}(G)}(S) / N_{\operatorname{Inn}(G)}(S)$ consisting of those classes that have a representative that restricts to the identity on $\mathcal{L}$. In particular, $\kappa_{G}$ is injective upon restriction to any Sylow p-subgroup of $\operatorname{Out}(G)$.

Proof. The group $A$ is the kernel of the composite $\mu_{G} \circ \kappa_{G}$, which is induced by restriction to $S$. By Theorem 1.2, the kernel of $\mu_{G}$ is either 1 or an elementary 2-group in the cases $p$ odd or $p=2$, respectively. So $\operatorname{ker}\left(\kappa_{G}\right)=O_{p^{\prime}}(A)$ by Theorem 5.1. The last statement follows immediately.

\begin{abstract}
Acknowledgements. The authors express their gratitude to the Institute of Mathematics at the University of Aberdeen for its hospitality during a research visit by the first author in 2017. The authors thank the Isaac Newton Institute for Mathematical Sciences, Cambridge, for support and hospitality during the programme groups, representations and applications where work on this article was undertaken. This work was supported by EPSRC grant no EP/R014604/1. Finally, the authors thank an anonymous referee who suggested a strengthening of Proposition 5.4 and made other comments that helped to improve the article.
\end{abstract}

\title{
Conflicts of Interest: None.
}

Financial support. GG was partially supported by a Simons Foundation Collaboration Grant. JL was partially supported by NSA Young Investigator Grant H98230-14-1-0312, Marie Curie Fellowship No. 707778, and NSF Grant DMS-1902152 during the preparation of this manuscript.

\section{References}

[AOV12] K. K. S. Andersen, B. Oliver and J. Ventura, 'Reduced, tame and exotic fusion systems', Proc. Lond. Math. Soc. (3) 105(1) (2012), 87-152.

[AKO11] M. Aschbacher, R. Kessar and B. Oliver, Fusion Systems in Algebra and Topology, London Mathematical Society Lecture Note Series, Vol. 391 (Cambridge University Press, Cambridge, UK, 2011).

[BLO03] C. Broto, R. Levi and B. Oliver, 'The homotopy theory of fusion systems', J. Amer. Math. Soc. 16(4) (2003), 779-856.

[BMO19] C. Broto, J. Møller and B. Oliver, 'Automorphisms of fusion systems of finite simple groups of Lie type', Mem. Amer. Math. Soc. 262(1267) (2019), 1-120. 
[Che13] A. Chermak, 'Fusion systems and localities', Acta Math. 211(1) (2013), 47-139.

[Che15] A. Chermak, 'Finite localities II', Preprint, 2015, arXiv:1505.08110.

[Che16] A. Chermak, 'Finite localities III', Preprint, 2016 arXiv:1610.06161.

[Cra11] D. A. Craven, 'Normal subsystems of fusion systems', J. Lond. Math. Soc. (2) 84(1) (2011), 137-158.

[Gla66a] G. Glauberman, 'Central elements in core-free groups', J. Algebra 4 (1966), 403-420.

[Gla66b] G. Glauberman, 'On the automorphism groups of a finite group having no non-identity normal subgroups of odd order', Math. Z. 93 (1966), 154-160.

[Gla68] G. Glauberman, 'Weakly closed elements of Sylow subgroups', Math. Z. 107 (1968), 1-20.

[GGLN20] G. Glauberman, R. Guralnick, J. Lynd and G. Navarro, 'Centers of Sylow subgroups and automorphisms', Israel J. Math. 240 (2020), 253-266.

[GL16] G. Glauberman and J. Lynd, 'Control of fixed points and existence and uniqueness of centric linking systems', Invent. Math. 206(2) (2016), 441-484.

[GLS98] D. Gorenstein, R. Lyons and R. Solomon, The Classification of the Finite Simple Groups. Number 3. Part I. Chapter A, Mathematical Surveys and Monographs, Vol. 40 (American Mathematical Society, Providence, RI, 1998).

[Gro82] F. Gross, 'Automorphisms which centralize a Sylow p-subgroup', J. Algebra 77(1) (1982), $202-233$.

[Hen19] E. Henke, 'Subcentric linking systems', Trans. Amer. Math. Soc. 371(5) (2019), 3325-3373.

[LO02] R. Levi and B. Oliver, 'Construction of 2-local finite groups of a type studied by Solomon and Benson', Geom. Topol. 6 (2002), 917-990.

[Oli04] B. Oliver, 'Equivalences of classifying spaces completed at odd primes', Math. Proc. Cambridge Philos. Soc. 137(2) (2004), 321-347.

[Oli06] B. Oliver, 'Equivalences of classifying spaces completed at the prime two', Mem. Amer. Math. Soc. 180(848) (2006), vi+102 pp.

[Oli13] B. Oliver, 'Existence and uniqueness of linking systems: Chermak's proof via obstruction theory', Acta Math. 211 (2013), no. 1, 141-175. MR 3118306

[OV07] B. Oliver and J. Ventura, 'Extensions of linking systems with p-group kernel', Math. Ann. 338(4) (2007), $983-1043$.

[Pui06] L. Puig, 'Frobenius categories', J. Algebra 303(1) (2006), 309-357. 